MATHEMATICS OF COMPUTATION

Volume 79, Number 269, January 2010, Pages 71-94

S 0025-5718(09)02251-0

Article electronically published on July 10, 2009

\title{
CONVERGENCE OF AN ENGQUIST-OSHER SCHEME FOR A MULTI-DIMENSIONAL TRIANGULAR SYSTEM OF CONSERVATION LAWS
}

\author{
G. M. COCLITE, S. MISHRA, AND N. H. RISEBRO
}

\begin{abstract}
We consider a multi-dimensional triangular system of conservation laws. This system arises as a model of three-phase flow in porous media and includes multi-dimensional conservation laws with discontinuous coefficients as a special case. The system is neither strictly hyperbolic nor symmetric. We propose an Engquist-Osher type scheme for this system and show that the approximate solutions generated by the scheme converge to a weak solution. Numerical examples are also presented.
\end{abstract}

\section{INTRODUCTION}

In this paper, we consider the $2 \times 2$ triangular system of conservation laws,

$$
\begin{aligned}
\partial_{t} u+\operatorname{div}(\mathbf{f}(u))=0, & \mathbf{x} \in \mathbb{R}^{d}, t>0, \\
\partial_{t} v+\operatorname{div}(\mathbf{g}(u, v))=0, & \mathbf{x} \in \mathbb{R}^{d}, t>0,
\end{aligned}
$$

with the initial condition

$$
u(\mathbf{x}, 0)=u_{0}(\mathbf{x}), v(\mathbf{x}, 0)=v_{0}(\mathbf{x}), \mathbf{x} \in \mathbb{R}^{d},
$$

where $u$ and $v$ are the unknowns, with the initial values $u_{0}$ and $v_{0}$, and the flux functions $\mathbf{f}=\left(f_{1}, f_{2}, \ldots, f_{d}\right)$ and $\mathbf{g}=\left(g_{1}, g_{2}, \ldots, g_{d}\right)$ are prescribed.

Note that the evolution of $u$ is independent of $v$, but that the evolution of $v$ depends on $u$. Writing (1.1), (1.2) in quasilinear form results in

$$
U_{t}+\sum_{i=1}^{d} A_{i} U_{x_{i}}=0
$$

where $U=\{u, v\}$ and the directional Jacobians are given by

$$
A_{i}=\left(\begin{array}{cc}
\frac{\partial f_{i}}{\partial u} & 0 \\
\frac{\partial g_{i}}{\partial u} & \frac{\partial g_{i}}{\partial v}
\end{array}\right) \text {. }
$$

Let $\mathbf{A}=\left\{A_{1}, A_{2}, \ldots, A_{d}\right\}$ and let $\mathbf{n}$ be a unit vector in $\mathbb{R}^{d}$. Then the matrix $\mathbf{A} \cdot \mathbf{n}$ is lower triangular. Hence, systems of the form (1.1), (1.2) are called triangular systems. Furthermore, the eigenvalues of the matrix $\mathbf{A} \cdot \mathbf{n}$ are real. Hence, the

Received by the editor July 30, 2008 and, in revised form, December 13, 2008.

2000 Mathematics Subject Classification. Primary 65L06, 35L65; Secondary 76S05.

The authors thank Kenneth H. Karlsen for many useful discussions. This research is supported in part by the Research Council of Norway. This paper was written as part of the international research program on Nonlinear Partial Differential Equations at the Centre for Advanced Study at the Norwegian Academy of Science and Letters in Oslo during the academic year 2008-09.

(C)2009 American Mathematical Society 
system is hyperbolic. Since the eigenvalues can coincide, the system is not strictly hyperbolic. Non-strictly hyperbolic systems are complicated to deal with even in the case of one space dimension.

In general, systems of conservation laws in several space dimensions are difficult to study and few rigorous results have been obtained. See however the book by Serre 2 for detailed references and theoretical results.

A special case of the above system occurs when we take $\mathbf{f}=0$. In this case, the system reduces to a multi-dimensional scalar conservation law with a spatially varying coefficient $u$ (which can be discontinuous). Scalar conservation laws with discontinuous coefficients arise in a wide variety of contexts including two-phase flows in heterogeneous porous media, as models of clarifier-thickener units and in traffic flow. In one spatial dimension such equations have been studied in several papers. An incomplete list includes [1, 3, 17, 9, 15, 17, 20, 21] and other references therein.

Scalar conservation laws with discontinuous coefficients in several space dimensions have not been correspondingly widely studied and the theory is not as welldeveloped as in the one-dimensional case. In [14, the authors considered a scalar conservation law in two space dimensions with discontinuous coefficients and obtained existence of weak solutions by showing that vanishing viscosity approximations converge. They used a modification of the compensated compactness approach in order to obtain compactness of approximate solutions. In [19, the author was able to treat a multi-dimensional scalar conservation law with discontinuous coefficients in both space and time. The author showed existence of weak solutions by proving compactness of approximations generated by smoothing the coefficients. The compactness technique in [19] uses the tool of $H$-measures extensively. We will adapt the compactness framework of [19] in this paper.

In one space dimension, the triangular system (1.1), (1.2) was considered in [13]. Existence of weak solutions was shown by constructing finite volume schemes and showing that the approximate solutions generated by these schemes are compact and converge to a weak solution. The compactness technique involved using discrete entropy inequalities and the compensated compactness framework.

Triangular systems occur in a variety of applications. We are motivated partly by a model of three-phase flow in porous media described briefly below.

A multi-dimensional three-phase flow model. Simulation of oil recovery processes involves models of three-phase flow in porous media. The three phases of interest are mostly oil, gas and water. Examples of three-phase flow include water flooding in the presence of gas, gas flooding and water alternating gas injections into a reservoir.

As a model, consider a homogeneous and isotropic porous medium. The phase saturations are given by $S_{\mathrm{o}}, S_{\mathrm{w}}$ and $S_{\mathrm{g}}$ for the oil, water and gas phases respectively. Mass conservation for each phase gives the following continuity equations:

$$
\phi\left(S_{l}\right)_{t}+\operatorname{div}\left(U_{l}\right)=q_{l}, \quad l=\mathrm{w}, \mathrm{o}, \mathrm{g},
$$

where $U_{l}$ is the phase flux of the $l$-th phase, $q_{l}$ is a source term and $\phi$ is the porosity of the medium, henceforth assumed to be equal to one. The phase flux for each phase is given by Darcy's law (see [4]) as

$$
U_{l}=\frac{-K k_{l}}{\mu_{l}}\left(\nabla p_{l}-\rho_{l} \tilde{g}\right),
$$


where $K$ is the absolute permeability, $k_{l}, \rho_{l}, p_{l}$, and $\mu_{l}$ are the relative permeability, density, pressure and viscosity of the $l$-th phase. The vector $\tilde{g}=\{0,0, g\}$ represents the gravitational force. The saturations satisfy the following condition:

$$
S_{\mathrm{o}}+S_{\mathrm{g}}+S_{\mathrm{w}}=1 .
$$

This implies that the gas and water phase saturations can be used as the unknowns. Furthermore, the phase pressures are also unknowns and have to be determined. We use the phase formulation for pressures (see 4 for details) and assume that the capillary pressures between the phases are zero. This assumption is valid when the flow is convection dominated, i.e., when the total flow rate is very high, and in this case we write $p=p_{l}$ for $l=\mathrm{w}, \mathrm{o}, \mathrm{g}$. Then introducing the total flux $U=U_{\mathrm{o}}+U_{\mathrm{g}}+U_{\mathrm{w}}$, phase mobilities $\lambda_{l}=\frac{k_{l}}{\mu_{l}}$ and total mobility $\lambda_{\mathrm{T}}=\lambda_{\mathrm{o}}+\lambda_{\mathrm{g}}+\lambda_{\mathrm{w}}$, we obtain the following equations for the total flux and pressure:

$$
\begin{aligned}
U & =-K \lambda_{\mathrm{T}}\left(\nabla p-\tilde{g}\left(\frac{\rho_{\mathrm{o}} \lambda_{\mathrm{o}}+\rho_{\mathrm{w}} \lambda_{\mathrm{w}}+\rho_{\mathrm{g}} \lambda_{\mathrm{g}}}{\lambda_{\mathrm{T}}}\right)\right), \\
\operatorname{div}(U) & =q_{\mathrm{o}}+q_{\mathrm{g}}+q_{\mathrm{w}} .
\end{aligned}
$$

The above equation for the pressure is elliptic and gives the total flux. The resulting equations for the phase saturations are given by

$$
\begin{gathered}
\left(S_{\mathrm{g}}\right)_{t}+F^{\mathrm{g}}\left(S_{\mathrm{g}}, S_{\mathrm{w}}, S_{\mathrm{o}}\right)_{x}+G^{\mathrm{g}}\left(S_{\mathrm{g}}, S_{\mathrm{w}}, S_{\mathrm{o}}\right)_{y}+H^{\mathrm{g}}\left(S_{\mathrm{g}}, S_{\mathrm{w}}, S_{\mathrm{o}}\right)_{z}=q_{\mathrm{g}}, \\
\left(S_{\mathrm{w}}\right)_{t}+F^{\mathrm{w}}\left(S_{\mathrm{g}}, S_{\mathrm{w}}, S_{\mathrm{o}}\right)_{x}+G^{\mathrm{w}}\left(S_{\mathrm{g}}, S_{\mathrm{w}}, S_{\mathrm{o}}\right)_{y}+H^{\mathrm{w}}\left(S_{\mathrm{g}}, S_{\mathrm{w}}, S_{\mathrm{o}}\right)_{z}=q_{\mathrm{w}} .
\end{gathered}
$$

Writing $U=\left\{u^{1}, u^{2}, u^{3}\right\}$ and assuming that the gravity acts in the $z$-direction, then the fluxes are given by

$$
\begin{aligned}
F^{\mathrm{g}} & =\frac{\lambda_{\mathrm{g}} u^{1}}{\lambda_{\mathrm{T}}}, \quad G^{\mathrm{g}}=\frac{\lambda_{\mathrm{g}} u^{2}}{\lambda_{\mathrm{T}}}, \\
F^{\mathrm{w}} & =\frac{\lambda_{\mathrm{w}} u^{1}}{\lambda_{\mathrm{T}}}, \quad G^{\mathrm{w}}=\frac{\lambda_{\mathrm{w}} u^{2}}{\lambda_{\mathrm{T}}}, \\
H^{\mathrm{g}} & =\frac{\lambda_{\mathrm{g}} u^{3}}{\lambda_{\mathrm{T}}}+K \frac{\lambda_{\mathrm{g}} \lambda_{\mathrm{w}}}{\lambda_{\mathrm{T}}}\left(\rho_{\mathrm{g}}-\rho_{\mathrm{w}}\right) g+K \frac{\lambda_{\mathrm{g}} \lambda_{\mathrm{o}}}{\lambda_{\mathrm{T}}}\left(\rho_{\mathrm{g}}-\rho_{\mathrm{o}}\right) g, \\
H^{\mathrm{w}} & =\frac{\lambda_{\mathrm{w}} u^{3}}{\lambda_{\mathrm{T}}}+K \frac{\lambda_{\mathrm{g}} \lambda_{\mathrm{w}}}{\lambda_{\mathrm{T}}}\left(\rho_{\mathrm{w}}-\rho_{\mathrm{g}}\right) g+K \frac{\lambda_{\mathrm{w}} \lambda_{\mathrm{o}}}{\lambda_{\mathrm{T}}}\left(\rho_{\mathrm{w}}-\rho_{\mathrm{o}}\right) g .
\end{aligned}
$$

Hence, the coupled system of equations (1.4), (1.5) and (1.6) model three-phase flow in a porous medium. This system couples an elliptic pressure equation with a hyperbolic saturation equation. These two equations have very different properties, and it is therefore common to use a spitting approach to obtain numerical solutions. In brief, this consists in fixing the saturation and solving the pressure equation (1.4), then fixing the pressure and solving the saturation equations (1.5) for a short time interval $\Delta t$, and repeating this process.

If we consider the saturation equations (1.5) with a given total flux $U$, the equations are a special case of a system of conservation laws in several space dimensions.

Despite this simplification, this system is quite complicated, both theoretically and computationally. Even in one space dimension, the above system is not necessarily hyperbolic and can contain elliptic regions. Therefore, as a further simplification, the following "reduced" model was proposed in [11] and was analysed in 13 in the one-dimensional case. This simplification is based on the observation that in many situations the mobility of the gas phase is much larger than that of 
the other phases. This means that the flux of gas is largely independent of whether the other phase is oil or water. As a consequence, we can make the approximations

$$
\begin{aligned}
& F^{\mathrm{g}}\left(S_{\mathrm{g}}, S_{\mathrm{w}}, S_{\mathrm{o}}\right)=\tilde{F}\left(S_{\mathrm{g}}, 1-S_{\mathrm{g}}\right)=\hat{F}\left(S_{\mathrm{g}}\right) \\
& G^{\mathrm{g}}\left(S_{\mathrm{g}}, S_{\mathrm{w}}, S_{\mathrm{o}}\right)=\tilde{G}\left(S_{\mathrm{g}}, 1-S_{\mathrm{g}}\right)=\hat{G}\left(S_{\mathrm{g}}\right), \\
& H^{\mathrm{g}}\left(S_{\mathrm{g}}, S_{\mathrm{w}}, S_{\mathrm{o}}\right)=\tilde{H}\left(S_{\mathrm{g}}, 1-S_{\mathrm{g}}\right)=\hat{H}\left(S_{\mathrm{g}}\right) .
\end{aligned}
$$

Assuming this, the system (1.5) reduces to the following system. The resulting equations for the phase saturations are given by

$$
\begin{array}{r}
\left(S_{\mathrm{g}}\right)_{t}+\hat{F}\left(S_{\mathrm{g}}\right)_{x}+\hat{G}\left(S_{\mathrm{g}}\right)_{y}+\hat{H}\left(S_{\mathrm{g}}\right)_{z}=q_{\mathrm{g}}, \\
\left(S_{\mathrm{w}}\right)_{t}+F^{\mathrm{w}}\left(S_{\mathrm{g}}, S_{\mathrm{w}}, S_{\mathrm{o}}\right)_{x}+G^{\mathrm{w}}\left(S_{\mathrm{g}}, S_{\mathrm{w}}, S_{\mathrm{o}}\right)_{y}+H^{\mathrm{w}}\left(S_{\mathrm{g}}, S_{\mathrm{w}}, S_{\mathrm{o}}\right)_{z}=q_{\mathrm{w}} .
\end{array}
$$

The above equation is a special case of the multi-dimensional triangular system (1.1), (1.2).

It is to be emphasized that although the assumption of independence of the gas phase is not valid for all fractional flow functions, there exists a large class of fractional flow functions for which this assumption appears to be reasonable. In view of the fact that this assumption makes the model simpler and much more tractable, we can use this "reduced" model in several situations. Nevertheless, we point out that a careful numerical study of this model (1.7) as an approximation to the full three-phase flow model needs to be carried out. An essential ingredient for this program is the development of efficient numerical schemes for (1.5) as well as for (1.7). We emphasize that the full three-phase flow model also includes the elliptic equation for the pressure. In this paper we concentrate on the saturation equations in the presence of a given total flux. In one space dimension, the pressure can be easily eliminated and the full model consists of the saturation equations only.

Our aim in this paper is to design an efficient numerical scheme to approximate solutions of the triangular system (1.1) and (1.2). We propose to use an adaptation of the Engquist-Osher scheme based on a staggered grid. The resulting approximate solutions are shown to converge to a weak solution of the triangular system. The proof of convergence is based on the framework developed in [19] and uses entropy inequalities extensively. We present some numerical experiments in order to demonstrate the robustness of the scheme. This numerical scheme can be used as the hyperbolic solver for three-phase flow models in several space dimensions, and can be coupled with a suitable elliptic solver for the pressure in order to design numerical codes for reservoir simulation.

In [5] we studied the same triangular system as in this paper, but as a limit of viscous regularizations. The basic compactness tools used in [5] were the same as in this paper, but obtaining the bounds necessary to use these compactness tools is much harder for a difference scheme than for the viscous approximations.

The rest of this paper is organized as follows: In Section 2, we present the mathematical framework used in this paper. The Engquist-Osher type scheme is proposed and analysed in Section 3 and numerical experiments are reported in Section 4. 


\section{MATHEMATICAL FRAMEWORK}

We start with the assumptions on the data and the flux functions,

(H.1) $\mathbf{f} \in C^{1}\left([-M, M] ; \mathbb{R}^{d}\right), \mathbf{g} \in C^{2}\left([-M, M]^{2} ; \mathbb{R}^{d}\right)$;

(H.2) $\partial_{v} \mathbf{g}(u, \pm M)=0$ for all $u$;

(H.3) $\partial_{u u}^{2} \mathbf{g}(\cdot, v)$ is Lipschitz continuous for $v \in[-M, M]$;

(H.4) $\mathbf{g}(u, \cdot)$ is genuinely non-linear for all $u$; i.e., for any unit vector $\mathbf{n}$, the map $v \in[-M, M] \mapsto \mathbf{g}(u, v) \cdot \mathbf{n}$ is not affine on any non-trivial interval for all $u$ and $\mathbf{n} \in \mathbb{R}^{d},|\mathbf{n}|=1$

(H.5) $u_{0}$ and $v_{0}$ are in $L^{\infty}\left(\mathbb{R}^{d}\right) \cap L^{1}\left(\mathbb{R}^{d}\right) \cap B V\left(\mathbb{R}^{d}\right)$

for some positive constant $M$.

As mentioned, we deal with weak solutions of (1.1) and (1.2) defined below.

Definition 2.1. We call the pair $(u, v)$ a weak solution of the Cauchy problem (1.1), (1.2) if

(D.1) $u$ and $v$ are in $L^{\infty}\left(\mathbb{R}^{d} \times \mathbb{R}^{+}\right)$;

(D.2) $u$ and $v$ satisfy (1.1), (1.2) in the sense of distributions on $\mathbb{R}^{d} \times \mathbb{R}^{+}$, i.e., the following identities,

$$
\begin{aligned}
& \int_{\mathbb{R}^{d} \times \mathbb{R}^{+}}\left(u \partial_{t} \varphi+\mathbf{f}(u) \operatorname{div}(\varphi)\right) d x d y d t+\int_{\mathbb{R}^{d}} u_{0}(x, y) \varphi(x, y, 0) d x d y=0, \\
& \int_{\mathbb{R}^{d} \times \mathbb{R}^{+}}\left(v \partial_{t} \varphi+\mathbf{g}(u, v) \operatorname{div}(\varphi)\right) d x d y d t+\int_{\mathbb{R}^{d}} v_{0}(x, y) \varphi(x, y, 0) d x d y=0,
\end{aligned}
$$

hold for each test function $\varphi$ smooth with compact support in $\mathbb{R}^{d} \times \mathbb{R}^{+}$;

(D.3) for each constant $k \in \mathbb{R}$ the inequality

$$
\partial_{t}|u-k|+\operatorname{div}(\operatorname{sign}(u-k)(\mathbf{f}(u)-\mathbf{f}(k))) \leq 0
$$

holds in the sense of distributions on $\mathbb{R}^{d} \times \mathbb{R}^{+}$.

As stated in the introduction, we will propose an Engquist-Osher type scheme in order to approximate the triangular system numerically. The convergence proof for the scheme is based on the following crucial lemma, which is an easy adaptation of a result by Panov [19, Theorem 5]. We call $(\eta, \mathbf{q})$ a convex entropy/entropy flux pair for (1.2) if $\eta$ is a convex function of $v$ and

$$
\partial_{v} \mathbf{q}(u, v)=\eta^{\prime}(v) \partial_{v} \mathbf{g}(u, v) .
$$

In particular, we have the Kružkov entropy/entropy flux pairs

$$
\eta_{0}(v)=|v-k|, \quad \mathbf{q}_{0}(u, v)=\operatorname{sign}(v-k)(g(u, v)-g(u, k)),
$$

where $k$ is an arbitrary constant.

Lemma 2.2. Let $u$ be the unique entropy solution of the Cauchy problem for the single conservation law (1.1), and let $\left\{v_{\nu}\right\}_{\nu>0}$ be a family of functions defined on $\mathbb{R}^{d} \times \mathbb{R}^{+}$. If $\left\{v_{\nu}\right\}$ is bounded in $L^{\infty}\left(\mathbb{R}^{d} \times \mathbb{R}^{+}\right)$, and

$$
\left\{\partial_{t} \eta_{0}\left(v_{\nu}\right)+\operatorname{div}\left(\mathbf{q}_{0}\left(u, v_{\nu}\right)\right)\right\}_{\nu>0}
$$

lies in a compact set of $H_{\mathrm{loc}}^{-1}\left(\mathbb{R}^{d} \times \mathbb{R}^{+}\right)$for all constants $k$, then there exists a sequence $\left\{\nu_{n}\right\}_{n \in \mathbb{N}}, \nu_{n} \rightarrow 0$ and a function $v \in L^{\infty}\left(\mathbb{R}^{d} \times \mathbb{R}^{+}\right)$such that

$$
v_{\nu_{n}} \rightarrow v \text { a.e. and in } L_{\mathrm{loc}}^{p}\left(\mathbb{R}^{d} \times \mathbb{R}^{+}\right), 1 \leq p<\infty \text {. }
$$

We also need the following useful technical lemma. 
Lemma 2.3 (see [18]). Let $\Omega$ be a bounded open subset of $\mathbb{R}^{d}, d \geq 2$. Suppose that the sequence $\left\{\mathcal{L}_{n}\right\}_{n \in \mathbb{N}}$ of distributions is bounded in $W^{-1, \infty}(\Omega)$. Suppose also that

$$
\mathcal{L}_{n}=\mathcal{L}_{1, n}+\mathcal{L}_{2, n}
$$

where $\left\{\mathcal{L}_{1, n}\right\}_{n \in \mathbb{N}}$ is in a compact subset of $H_{\text {loc }}^{-1}(\Omega)$ and $\left\{\mathcal{L}_{2, n}\right\}_{n \in \mathbb{N}}$ is in a bounded subset of $\mathcal{M}_{\mathrm{loc}}(\Omega)$. Then $\left\{\mathcal{L}_{n}\right\}_{n \in \mathbb{N}}$ is in a compact subset of $H_{\mathrm{loc}}^{-1}(\Omega)$.

Throughout the paper, we use the notation $C_{X}$ to indicate a constant depending on the quantity $X$ (only).

\section{The Engquist-Osher method}

Now we shall analyze the Engquist-Osher method for (1.1) and (1.2). In order to simplify the notation, we restrict ourselves to two spatial dimensions, i.e., $d=2$, but the generalization to arbitrary $d$ is straightforward. In this case,

$$
\mathbf{f}(u)=\left(f_{1}(u), f_{2}(u)\right) \quad \text { and } \quad \mathbf{g}(u, v)=\left(g_{1}(u, v), g_{2}(u, v)\right) .
$$

We shall use a staggered version of the Engquist-Osher scheme, in which $u$ and $v$ are discretized on grids which are staggered and rotated with respect to each other. Set

$$
\tilde{\mathbf{f}}=\frac{1}{\sqrt{2}}\left(\begin{array}{cc}
1 & -1 \\
1 & 1
\end{array}\right) \mathbf{f} \quad \text { and } \quad \tilde{\mathbf{x}}=\frac{1}{\sqrt{2}}\left(\begin{array}{cc}
1 & -1 \\
1 & 1
\end{array}\right) \mathbf{x} .
$$

Then we observe that $u$ is (also) the entropy solution of

$$
\partial_{t} u+\widetilde{\operatorname{div}}(\tilde{\mathbf{f}})=0, \quad u(\tilde{\mathbf{x}}, 0)=u_{0}(\tilde{\mathbf{x}})
$$

where $\widetilde{\operatorname{div}} \tilde{\mathbf{f}}=\left(\partial_{\tilde{x}}, \partial_{\tilde{y}}\right) \cdot \tilde{\mathbf{f}}$. We shall consider discrete versions of (3.1) and (1.2).

For a scalar function $f_{\ell}(u)$, the Engquist-Osher flux is defined as

$$
f_{\ell}^{\mathrm{EO}}(a, b)=\int_{0}^{a}\left(f_{\ell}^{\prime}(s) \vee 0\right) d s+\int_{0}^{b}\left(f_{\ell}^{\prime}(s) \wedge 0\right) d s+f_{\ell}(0),
$$

where we use the notation $(a \wedge b)=\min \{a, b\}$ and $(a \vee b)=\max \{a, b\}$.

We use the same grid spacing in both directions $\Delta x=\Delta y=h>0$ and a uniform time step $\Delta t>0$. Set $x_{i}=i h$ and $y_{j}=j h$ for integers $i$ and $j$, and $x_{i+1 / 2}=x_{i}+h / 2, y_{j+1 / 2}=y_{j}+h / 2$ and $t^{n}=n \Delta t$. Let the squares $\mathcal{I}_{i, j+1 / 2}$ and $\mathcal{I}_{i+1 / 2, j}$ be defined by

$$
\begin{aligned}
& \mathcal{I}_{i, j+1 / 2}=\left\{(x, y)|| x-x_{i}|+| y-y_{j+1 / 2} \mid<h / 2\right\}, \\
& \mathcal{I}_{i+1 / 2, j}=\left\{(x, y)|| x-x_{i+1 / 2}|+| y-y_{j} \mid<h / 2\right\} .
\end{aligned}
$$

For later use, we also define the cells

$$
\mathcal{I}_{i, j}=\left\{(x, y) \mid\left(\left|x-x_{i}\right| \vee\left|y-y_{j}\right|\right)<h / 2\right\} .
$$


With $\lambda=\Delta t / h$, the Engquist-Osher scheme for (3.1) reads

$$
\begin{aligned}
u_{i, j+1 / 2}^{n+1}= & u_{i, j+1 / 2}^{n}-\sqrt{2} \lambda\left(\tilde{f}_{1}^{\mathrm{EO}}\left(u_{i, j+1 / 2}^{n}, u_{i+1 / 2, j}^{n}\right)\right. \\
& -\tilde{f}_{1}^{\mathrm{EO}}\left(u_{i-1 / 2, j+1}^{n}, u_{i, j+1 / 2}^{n}\right) \\
& \left.+\tilde{f}_{2}^{\mathrm{EO}}\left(u_{i, j+1 / 2}^{n}, u_{i+1 / 2, j+1}^{n}\right)-\tilde{f}_{2}^{\mathrm{EO}}\left(u_{i-1 / 2, j}^{n}, u_{i, j+1 / 2}^{n}\right)\right), \\
u_{i+1 / 2, j}^{n+1}= & u_{i+1 / 2, j}^{n}-\sqrt{2} \lambda\left(\tilde{f}_{1}^{\mathrm{EO}}\left(u_{i+1 / 2, j}^{n}, u_{i+1, j-1 / 2}^{n}\right)\right. \\
& -\tilde{f}_{1}^{\mathrm{EO}}\left(u_{i, j+1 / 2}^{n}, u_{i+1 / 2, j}^{n}\right) \\
& \left.+\tilde{f}_{2}^{\mathrm{EO}}\left(u_{i+1 / 2, j}^{n}, u_{i+1, j+1 / 2}^{n}\right)-\tilde{f}_{2}^{\mathrm{EO}}\left(u_{i, j-1 / 2}^{n}, u_{i+1 / 2, j}^{n}\right)\right),
\end{aligned}
$$

for integers $i$ and $j$ and $n \geq 0$. The scheme is initiated by setting

$$
u_{i, j+1 / 2}^{0}=\frac{2}{h^{2}} \iint_{\mathcal{I}_{i, j+1 / 2}} u_{0}(\mathbf{x}) d \mathbf{x}, \quad u_{i+1 / 2, j}^{0}=\frac{2}{h^{2}} \iint_{\mathcal{I}_{i+1 / 2, j}} u_{0}(\mathbf{x}) d \mathbf{x} .
$$

The relation between $h$ and $\Delta t$ is such that the CFL-condition

$$
4 \sqrt{2} \lambda\left\|\tilde{\mathbf{f}}^{\prime}\right\|_{L^{\infty}} \leq 1
$$

holds. Let $u_{h}$ be defined as the piecewise constant function

$$
u_{h}(x, y, t)=\left\{\begin{array}{ll}
u_{i+1 / 2, j}^{n}, & \text { for }(x, y) \in \mathcal{I}_{i+1 / 2, j} \\
u_{i, j+1 / 2}^{n}, & \text { for }(x, y) \in \mathcal{I}_{i, j+1 / 2},
\end{array} \quad \text { for } t \in\left[t^{n}, t^{n+1}\right), n \geq 0 .\right.
$$

We have that $\lim _{h \rightarrow 0} u_{h}$ is the unique entropy solution of (1.1). We list some useful properties of $u_{h}$ in the next lemma [10].

Lemma 3.1. Assume that the CFL condition (3.3) holds. Then for each $h>0$ we have that

(a) $-M \leq u_{h}(x, y, t) \leq M$, for all $(x, y)$ and $t \geq 0$.

(b) For $n \geq 0$ the functions

$$
\begin{aligned}
n & \mapsto \frac{h^{2}}{2} \sum_{i, j}\left|u_{i+1 / 2, j}^{n}\right|+\left|u_{i, j+1 / 2}^{n}\right|, \\
n & \mapsto \sum_{i, j}\left|u_{i, j+1 / 2}^{n}-u_{i-1 / 2, j}^{n}\right|+\left|u_{i+1 / 2, j}^{n}-u_{i, j-1 / 2}^{n}\right|, \\
n & \mapsto \sum_{i, j}\left|u_{i+1 / 2, j}^{n+1}-u_{i+1 / 2, j}^{n}\right|+\left|u_{i, j+1 / 2}^{n+1}-u_{i, j+1 / 2}^{n}\right|
\end{aligned}
$$

are non-increasing. In particular this means that the family $\left\{u_{h}\right\}_{h>0}$ is (uniformly in $h$ ) bounded in $L^{\infty}\left(\mathbb{R}^{+} ; L^{1}\left(\mathbb{R}^{2}\right)\right) \cap B V\left(\mathbb{R}^{2} \times \mathbb{R}^{+}\right)$.

(c) The sequence $\left\{u_{h}\right\}_{h>0}$ converges to the unique entropy solution $u$ of the conservation law (1.1), i.e.,

$$
u_{h} \rightarrow u \text { a.e. and in } L_{\mathrm{loc}}^{p}\left(\mathbb{R}^{2} \times \mathbb{R}^{+}\right), 1 \leq p<\infty .
$$


When we formulate the Engquist-Osher scheme for $v$, we need EO-fluxes for functions of two variables $g_{\ell}(u, v)$. We define this as

$$
g_{\ell}^{\mathrm{EO}}(u, a, b)=\int_{0}^{a}\left(\partial_{v} g_{\ell}(u, s) \vee 0\right) d s+\int_{0}^{b}\left(\partial_{v} g_{\ell}(u, s) \wedge 0\right) d s+g_{\ell}(u, 0) ;
$$

i.e., the first variable is regarded as a parameter. The EO-flux is Lipschitz continuous in all variables and has the useful monotonicity properties,

$$
\partial_{a} g_{\ell}^{\mathrm{EO}}(u, a, b) \geq 0, \partial_{b} g_{\ell}^{\mathrm{EO}}(u, a, b) \leq 0,
$$

and satisfies the bounds

$$
\left|\partial_{a} g_{\ell}^{\mathrm{EO}}(u, a, b)\right| \leq\left\|\partial_{v} g_{\ell}\right\|_{L^{\infty}},\left|\partial_{b} g_{\ell}^{\mathrm{EO}}(u, a, b)\right| \leq\left\|\partial_{v} g_{\ell}\right\|_{L^{\infty}} .
$$

The Engquist-Osher scheme corresponding to (1.2) reads

$$
\begin{aligned}
v_{i, j}^{n+1}=v_{i, j}^{n}-\lambda & g_{1}^{\mathrm{EO}}\left(u_{i+1 / 2, j}^{n}, v_{i, j}^{n}, v_{i+1, j}^{n}\right)-g_{1}^{\mathrm{EO}}\left(u_{i-1 / 2, j}^{n}, v_{i-1, j}^{n}, v_{i, j}^{n}\right) \\
& \left.+g_{2}^{\mathrm{EO}}\left(u_{j+1 / 2, i}^{n}, v_{i, j}^{n}, v_{i, j+1}^{n}\right)-g_{2}^{\mathrm{EO}}\left(u_{j-1 / 2, i}^{n}, v_{i, j-1}^{n}, v_{i, j}^{n}\right)\right),
\end{aligned}
$$

with initial values

$$
v_{i, j}^{0}=\frac{1}{h^{2}} \iint_{\mathcal{I}_{i, j}} v_{0}(\mathbf{x}) d \mathbf{x}
$$

The reason for using this "staggered" scheme is that we want to have $u$ constant across discontinuities in $v$. This enables the use of simple scalar numerical flux functions such as the Engquist-Osher flux. Figure 1 shows the location of the discrete variables and the grids used for $u$ and $v$. We shall prove the following convergence result.

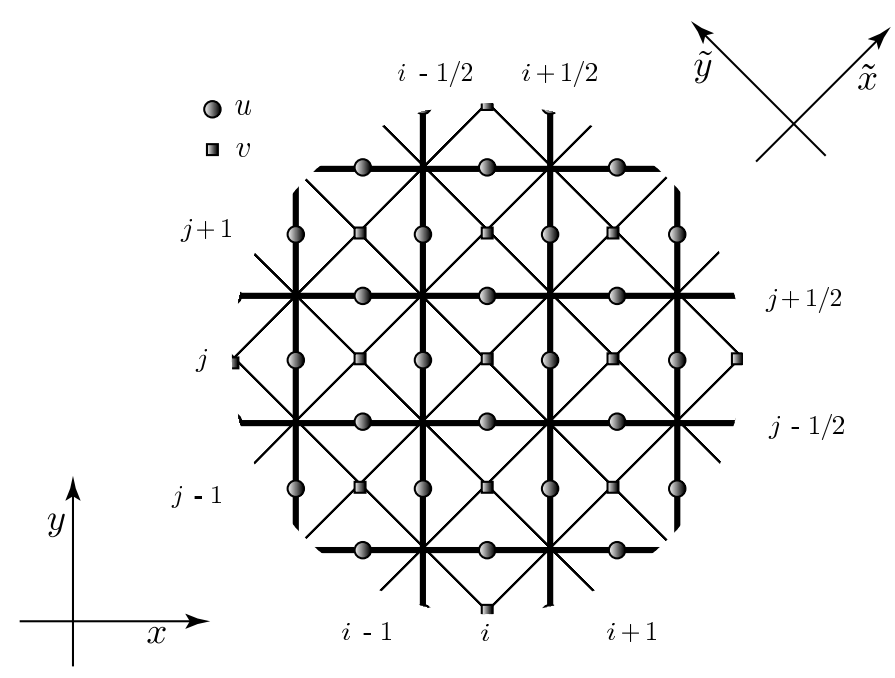

Figure 1. The locations of the grid cells for $u$ and $v$. 
Theorem 3.2. If the CFL-condition

$$
\lambda \leq \min \left\{\frac{1}{4 \sqrt{2}\left\|\tilde{\mathbf{f}}^{\prime}\right\|_{L^{\infty}}}, \frac{1}{4\left\|\partial_{v} \mathbf{g}\right\|_{L^{\infty}}}\right\}
$$

holds, then the scheme (3.2)-(3.5) produces a sequence which converges to a weak solution of the Cauchy problem (1.1) -(1.2) in $L_{\text {loc }}^{p}\left(\mathbb{R}^{2} \times \mathbb{R}^{+}\right), 1 \leq p<\infty$.

Proof. The theorem follows from Lemmas 2.2, 3.4 and 3.5.

If the CFL-condition

$$
4 \lambda\left\|\partial_{v} \mathbf{g}\right\|_{L^{\infty}} \leq 1
$$

holds, the scheme is monotone in the sense described below. We define the piecewise constant functions

$$
v^{n}(\mathbf{x})=v_{i, j}^{n} \quad \text { for } \mathbf{x} \in \mathcal{I}_{i, j} \quad \text { and } \quad v_{h}(\mathbf{x}, t)=v^{n}(\mathbf{x}) \quad \text { for } t \in\left[t^{n}, t^{n+1}\right) .
$$

We can write (3.5) as

$$
v_{i, j}^{n+1}=F_{i, j}^{n}\left(v_{i, j}^{n}, v_{i-1, j}^{n}, v_{i+1, j}^{n}, v_{i, j-1}^{n}, v_{i, j+1}^{n}\right) \quad \text { or } \quad v^{n+1}=F^{n}\left(v^{n}\right),
$$

and it is easy to see that the CFL-condition (3.8) implies that $F_{i, j}^{n}$ is non-decreasing in all arguments. Furthermore, by Assumption (H.2)

$$
F_{i, j}^{n}( \pm M, \pm M, \pm M, \pm M, \pm M)= \pm M \text {. }
$$

Thus if $v^{n}(\mathbf{x}) \in[-M, M]$, then also $v^{n+1} \in[-M, M]$. At this point we recall the useful result by Crandall and Tartar.

Lemma 3.3 (Crandall and Tartar [6]). Let $(\Omega, \mu)$ be some measure space and let $D$ be a subset of $L^{1}(\Omega)$ such that if $u$ and $v$ are in $D$, then so is $(u \vee v)$. Let $T$ be a map $D \mapsto D$ such that

$$
\int_{\Omega} T(u) d \mu=\int_{\Omega} u d \mu \quad \text { for all } u \in D .
$$

Then the following statements, valid for all $u$ and $v$ in $D$, are equivalent:

(i) If $u \leq v$, then $T(u) \leq T(v)$.

(ii) $\int_{\Omega}((T(u)-T(v)) \vee 0) d \mu \leq \int_{\Omega}((u-v) \vee 0) d \mu$.

(iii) $\int_{\Omega}|T(u)-T(v)| d \mu \leq \int_{\Omega}|u-v| d \mu$.

We can use this lemma for the mapping $v \mapsto F^{n}(v)$, where $D$ is the subset of $L^{1}\left(\mathbb{R}^{2}\right)$ consisting of functions that are constant on $\mathcal{I}_{i, j}$. Then the monotonicity of $F_{i, j}^{n}$ implies that if $v$ and $\tilde{v}$ are in $D$, then we have

$$
\tilde{v} \leq v \Rightarrow F^{n}(\tilde{v}) \leq F^{n}(v) .
$$

By (iii) we then find

$$
h^{2} \sum_{i, j}\left|F_{i, j}^{n}\left(v^{n}\right)-F_{i, j}^{n}(0)\right| \leq h^{2} \sum_{i, j}\left|v_{i, j}^{n}\right|
$$

or

$$
h^{2} \sum_{i, j}\left|v_{i, j}^{n+1}\right| \leq h^{2} \sum_{i, j}\left|v_{i, j}^{n}\right|+C \Delta t\left|u_{0}\right|_{B V\left(\mathbb{R}^{2}\right)},
$$

where $C$ is a constant depending on $\mathbf{g}$, but not on $\Delta t$. 
Next, to save space and typing efforts, we introduce the notation $I=(i, j)$, $e_{1}=(1,0), e_{2}=(0,1)$ and

$$
D_{\ell} \alpha_{I}=\alpha_{I+e_{\ell}}-\alpha_{I}
$$

Furthermore, we shall apply $D_{\ell}$ to functions of the variables " $u$ " and " $v$ ". Let $D_{i}^{u}$ be the difference only in the $u$ variable and $D_{i}^{v}$ only in the $v$ variable, so that

$$
\begin{aligned}
D_{\ell}^{u} \alpha\left(u_{I}, v_{I}, w_{I}\right) & =\alpha\left(u_{I+e_{\ell}}, v_{I}, w_{I}\right)-\alpha\left(u_{I}, v_{I}, w_{I}\right), \\
D_{\ell}^{v} \alpha\left(u_{I}, v_{I-e_{\ell}}, v_{I}\right) & =\alpha\left(u_{I}, v_{I}, v_{I+e_{\ell}}\right)-\alpha\left(u_{I}, v_{I-e_{\ell}}, v_{I}\right), \\
D_{\ell} \alpha\left(u_{I}, v_{I}\right) & =D_{\ell}^{u} \alpha\left(u_{I}, v_{I}\right)+D_{\ell}^{v} \alpha\left(u_{I+e_{\ell}}, v_{I}\right) \\
& =D_{\ell}^{v} \alpha\left(u_{I}, v_{I}\right)+D_{\ell}^{u} \alpha\left(u_{I}, v_{I+e_{\ell}}\right) .
\end{aligned}
$$

We can also use (iii) with $u=v_{I}^{n}$ and $v=v_{I}^{n-1}$ to conclude that

$$
\begin{aligned}
h^{2} \sum_{I}\left|v_{I}^{n+1}-v_{I}^{n}\right| & \leq h^{2} \sum_{I}\left|v_{I}^{n}-v_{I}^{n-1}\right| \\
& \leq h^{2} \sum_{I}\left|v_{I}^{1}-v_{I}^{0}\right| \\
& \leq h^{2} \lambda \sum_{I} \sum_{\ell}\left|D_{\ell} g_{\ell}^{\mathrm{EO}}\left(u_{I-e_{\ell} / 2}^{0}, v_{I-e_{\ell}}^{0}, v_{I}^{0}\right)\right| \\
& \leq C \Delta t
\end{aligned}
$$

if $v_{0}$ and $u_{0}$ are of bounded variation 1 This leads to a bound on the discrete divergence of the numerical flux,

$$
h^{2} \sum_{I}\left|\sum_{\ell} \frac{1}{h} D_{\ell} g_{\ell}^{\mathrm{EO}}\left(u_{I-e_{\ell} / 2}^{n}, v_{I-e_{\ell}}^{n}, v_{I}^{n}\right)\right| \leq C .
$$

Using the " $D \ell$ " notation we can write (3.5) as

$$
\begin{aligned}
v_{I}^{n+1} & =v_{I}^{n}-\lambda \sum_{\ell} D_{\ell} g_{\ell}^{\mathrm{EO}}\left(u_{I-e_{\ell} / 2}^{n}, v_{I-e_{\ell}}^{n}, v_{I}^{n}\right) \\
& =\underbrace{v_{I}^{n}-\lambda \sum_{\ell} D_{\ell}^{v} g_{\ell}^{\mathrm{EO}}\left(u_{I+e_{\ell} / 2}^{n}, v_{I-e_{\ell} / 2}^{n}, v_{I}^{n}\right)}_{w_{I}^{n}}-\lambda \sum_{\ell} D_{\ell}^{u} g_{\ell}^{\mathrm{EO}}\left(u_{I-e_{\ell}}^{n}, v_{I-e_{\ell}}^{n}, v_{I}^{n}\right) .
\end{aligned}
$$

Let $\eta$ be a convex entropy, and let $q_{\ell}^{\mathrm{EO}}(u, a, b)$ denote the associated numerical entropy flux

$$
q_{\ell}^{\mathrm{EO}}(u, a, b)=\int_{0}^{a} \eta^{\prime}(s)\left(\partial_{v} g_{\ell}(u, s) \vee 0\right) d s+\int_{0}^{b} \eta^{\prime}(s)\left(\partial_{v} g_{\ell}(u, s) \wedge 0\right) d s .
$$

Since the scheme is monotone we get

$$
\eta\left(w_{I}^{n}\right) \leq \eta\left(v_{I}^{n}\right)-\lambda \sum_{\ell} D_{\ell}^{v} q_{\ell}^{\mathrm{EO}}\left(u_{I+e_{\ell} / 2}^{n}, v_{I-e_{\ell}}^{n}, v_{I}^{n}\right),
$$

while by the convexity of $\eta$ and the definition of $w_{I}^{n}$, see (3.11),

$$
\eta\left(w_{I}^{n}\right) \geq \eta\left(v_{I}^{n+1}\right)+\eta^{\prime}\left(v_{I}^{n+1}\right) \lambda \sum_{\ell} D_{\ell}^{u} g_{\ell}^{\mathrm{EO}}\left(u_{I-e_{\ell} / 2}^{n}, v_{I-e_{\ell}}^{n}, v_{I}^{n}\right) .
$$

\footnotetext{
${ }^{1}$ This is only a sufficient condition. We just need to assume that the initial discrete divergence is bounded, i.e., $\sum_{I}\left|\sum_{\ell} D_{\ell} g_{\ell, I}^{\mathrm{EO}}\left(u_{I-e_{\ell} / 2}^{0}, v_{I-e_{\ell}}^{0}, v_{I}^{0}\right)\right| \leq C$.
} 
Using (3.11), (3.12) and (3.13), we arrive at the following estimate:

$$
\begin{gathered}
\eta\left(v_{I}^{n+1}\right) \leq \eta\left(v_{I}^{n}\right)-\lambda \sum_{\ell} D_{\ell} q_{\ell}^{\mathrm{EO}}\left(u_{I-e_{\ell} / 2}^{n}, v_{I-e_{\ell}}^{n}, v_{I}^{n}\right) \\
-\lambda \sum_{\ell} D_{\ell}^{u}\left(\eta^{\prime}\left(v_{I}^{n+1}\right) g_{\ell}^{\mathrm{EO}}\left(u_{I-e_{\ell} / 2}^{n}, v_{I-e_{\ell}}^{n}, v_{I}^{n}\right)\right. \\
\left.-q_{\ell}^{\mathrm{EO}}\left(u_{I-e_{\ell} / 2}^{n}, v_{I-e_{\ell}}^{n}, v_{I}^{n}\right)\right) .
\end{gathered}
$$

Since $v_{h}$ is bounded, and both $g_{\ell}^{\mathrm{EO}}$ and $q_{\ell}^{\mathrm{EO}}$ are Lipschitz continuous, and $u_{h}$ is of bounded variation we get the bound for non-negative $\eta$ :

$$
\left\|\eta\left(v_{h}(\cdot, t)\right)\right\|_{L^{1}\left(\mathbb{R}^{2}\right)} \leq\left\|\eta\left(v_{0}\right)\right\|_{L^{1}\left(\mathbb{R}^{2}\right)}+C t\left|u_{0}\right|_{B V\left(\mathbb{R}^{2}\right)},
$$

for some constant $C$ which does not depend on $h$. Choosing $\eta(v)=v^{2}$ we get the $L^{2}$ estimate

$$
\left\|v_{h}(\cdot, t)\right\|_{L^{2}\left(\mathbb{R}^{2}\right)}^{2} \leq\left\|v_{0}\right\|_{L^{2}\left(\mathbb{R}^{2}\right)}^{2}+C t\left|u_{0}\right|_{B V\left(\mathbb{R}^{2}\right)} .
$$

We can actually get a stronger estimate by multiplying the scheme (3.11) by $\eta^{\prime}\left(v_{I}^{n+1}\right)$ and using the Taylor expansion

$$
\eta^{\prime}\left(v_{I}^{n+1}\right)\left(v_{I}^{n+1}-v_{I}^{n}\right)=\eta\left(v_{I}^{n+1}\right)-\eta\left(v_{I}^{n}\right)+\frac{1}{2} \eta^{\prime \prime}\left(\xi_{I}^{n+1 / 2}\right)\left(v_{I}^{n+1}-v_{I}^{n}\right)^{2},
$$

where $\xi_{I}^{n+1 / 2}$ is an intermediate value. Doing this gives

$$
\begin{aligned}
\eta\left(v_{I}^{n+1}\right)-\eta\left(v_{I}^{n}\right)+ & \frac{1}{2} \eta^{\prime \prime}\left(\xi_{I}^{n+1 / 2}\right)\left(v_{I}^{n+1}-v_{I}^{n}\right)^{2} \\
= & -\lambda \eta^{\prime}\left(v_{I}^{n+1}\right) \sum_{\ell} D_{\ell} g_{\ell}^{\mathrm{EO}}\left(u_{I-e_{\ell} / 2}^{n}, v_{I-e_{\ell}}^{n}, v_{I}^{n}\right) \\
= & -\lambda \eta^{\prime}\left(v_{I}^{n}\right) \sum_{\ell} D_{\ell} g_{\ell}^{\mathrm{EO}}\left(u_{I-e_{\ell} / 2}^{n}, v_{I-e_{\ell}}^{n}, v_{I}^{n}\right) \\
& \quad-\lambda\left(\eta^{\prime}\left(v_{I}^{n+1}\right)-\eta^{\prime}\left(v_{I}^{n}\right)\right) \sum_{\ell} D_{\ell} g_{\ell}^{\mathrm{EO}}\left(u_{I-e_{\ell} / 2}^{n}, v_{I-e_{\ell}}^{n}, v_{I}^{n}\right) .
\end{aligned}
$$

We follow [16] and define

$$
g^{-}(u, v)=\int_{0}^{v}\left(\partial_{v} g(u, s) \wedge 0\right) d s, \quad g^{+}(u, v)=\int_{0}^{v}\left(\partial_{v} g(u, s) \vee 0\right) d s,
$$

and introduce the functions

$$
\mathcal{G}_{\ell}^{ \pm}(u, v)=\int_{0}^{v} \eta^{\prime}(s) \partial_{v} g_{\ell}^{ \pm}(u, s) d s .
$$

Integration by parts gives

$$
\begin{aligned}
\mathcal{G}_{\ell}^{ \pm}(u, b)-\mathcal{G}_{\ell}^{ \pm}(u, a)= & \eta^{\prime}(b) \\
& \left(g_{\ell}^{ \pm}(u, b)-g_{\ell}^{ \pm}(u, a)\right) \\
& -\int_{a}^{b} \eta^{\prime \prime}(s)\left(g_{\ell}^{ \pm}(u, s)-g_{\ell}^{ \pm}(u, a)\right) d s .
\end{aligned}
$$


Therefore,

$$
\begin{aligned}
\eta^{\prime}\left(v_{I}^{n}\right) & D_{\ell}^{v} g_{\ell}^{-}\left(u_{I-e_{\ell} / 2}^{n}, v_{I}^{n}\right)=D_{\ell} \mathcal{G}_{\ell}^{-}\left(u_{I-e_{\ell} / 2}^{n}, v_{I}^{n}\right)-D_{\ell}^{u} \mathcal{G}_{\ell}^{-}\left(u_{I-e_{\ell} / 2}^{n}, v_{I+e_{\ell}}^{n}\right) \\
& -\int_{v_{I+e_{\ell}}^{n}}^{v_{I}^{n}} \eta^{\prime \prime}(s)\left(g_{\ell}^{-}\left(u_{I-e_{\ell} / 2}^{n}, s\right)-g_{\ell}^{-}\left(u_{I-e_{\ell} / 2}^{n}, v_{I+e_{\ell}}^{n}\right)\right) d s \\
\eta^{\prime}\left(v_{I}^{n}\right) & D_{\ell}^{v} g_{\ell}^{+}\left(u_{I-e_{\ell} / 2}^{n}, v_{I-e_{\ell}}^{n}\right)=D_{\ell} \mathcal{G}_{\ell}^{+}\left(u_{I-e_{\ell} / 2}^{n}, v_{I-e_{\ell}}^{n}\right)-D_{\ell}^{u} \mathcal{G}_{\ell}^{+}\left(u_{I-e_{\ell} / 2}^{n}, v_{I}^{n}\right) \\
& +\int_{v_{I-e_{\ell}}^{n}}^{v_{I}^{n}} \eta^{\prime \prime}(s)\left(g_{\ell}^{+}\left(u_{I-e_{\ell} / 2}^{n}, s\right)-g_{1}^{+}\left(u_{I-e_{\ell} / 2}^{n}, v_{I-e_{\ell}}^{n}\right)\right) d s
\end{aligned}
$$

Hence,

$$
\begin{aligned}
\eta^{\prime}\left(v_{I}^{n}\right) & D_{\ell} g_{\ell}^{\mathrm{EO}}\left(u_{I-e_{\ell} / 2}^{n}, v_{I-e_{\ell}}^{n}, v_{I}^{n}\right)=D_{\ell} q_{\ell}^{\mathrm{EO}}\left(u_{I-e_{\ell} / 2}^{n}, v_{I-e_{\ell}}^{n}, v_{I}^{n}\right) \\
& -D_{\ell}^{u}\left(\eta^{\prime}\left(v_{I}^{n}\right) g_{\ell}^{\mathrm{EO}}\left(u_{I-e_{\ell} / 2}^{n}, v_{I}^{n}, v_{I+e_{\ell}}^{n}\right)-q_{\ell}^{\mathrm{EO}}\left(u_{I-e_{\ell} / 2}^{n}, v_{I}^{n}, v_{I+e_{\ell}}^{n}\right)\right) \\
& -\underbrace{\int_{v_{I+e_{\ell}}^{n}}^{v_{I}^{n}} \eta^{\prime \prime}(s)\left(g_{\ell}^{-}\left(u_{I-e_{\ell} / 2}^{n}, s\right)-g_{\ell}^{-}\left(u_{I-e_{\ell} / 2}^{n}, v_{I+e_{\ell}}^{n}\right)\right) d s}_{\Theta_{I, \ell}^{-}} \\
& +\underbrace{\int_{v_{I-e_{\ell}}^{n} \eta^{\prime \prime}}^{v_{I}^{n}}(s)\left(g_{\ell}^{+}\left(u_{I-e_{\ell} / 2}^{n}, s\right)-g_{1}^{+}\left(u_{I-e_{\ell} / 2}^{n}, v_{I-e_{\ell}}^{n}\right)\right) d s}_{\Theta_{I, \ell}^{+}}
\end{aligned}
$$

Since $g_{\ell}^{-}$is decreasing in $v$, and $g_{\ell}^{+}$is increasing in $v, \Theta_{I, \ell}^{-}, \Theta_{I, \ell}^{+} \geq 0$. Thus (3.17) can be written as

$$
\begin{gathered}
\eta\left(v_{I}^{n+1}\right)-\eta\left(v_{I}^{n}\right)+\frac{1}{2} \eta^{\prime \prime}\left(\xi_{I}^{n+1 / 2}\right)\left(v_{I}^{n+1}-v_{I}^{n}\right)^{2}+\lambda \sum_{\ell}\left(\Theta_{I, \ell}^{+}+\Theta_{I, \ell}^{-}\right) \\
=-\lambda \sum_{\ell} D_{\ell} q_{\ell}^{\mathrm{EO}}\left(u_{I-e_{\ell} / 2}^{n}, v_{I-e_{\ell}}^{n}, v_{I}^{n}\right) \\
-\lambda\left(\eta^{\prime}\left(v_{I}^{n+1}\right)-\eta^{\prime}\left(v_{I}^{n}\right)\right) \sum_{\ell} D_{\ell} g_{\ell}^{\mathrm{EO}}\left(u_{I-e_{\ell} / 2}^{n}, v_{I-e_{\ell}}^{n}, v_{I}^{n}\right) \\
-\lambda \sum_{\ell} D_{\ell}^{u}\left(\eta^{\prime}\left(v_{I}^{n}\right) g_{\ell}^{\mathrm{EO}}\left(u_{I-e_{\ell} / 2}^{n}, v_{I}^{n}, v_{I+e_{\ell}}^{n}\right)\right. \\
\left.-q_{\ell}^{\mathrm{EO}}\left(u_{I-e_{\ell} / 2}^{n}, v_{I}^{n}, v_{I+e_{\ell}}^{n}\right)\right) .
\end{gathered}
$$

Now we can multiply this by $h^{2}$, sum over $n \in\{0, \ldots, N-1\}$ and $I \in \mathbb{Z}^{2}$, and use the divergence bound (3.10) and the fact that $u_{h}$ has bounded variation to get 


$$
\begin{aligned}
h^{2} \sum_{I} & \eta\left(v_{I}^{N}\right)+\frac{1}{2} h^{2} \sum_{n, I} \eta^{\prime \prime}\left(\xi_{I}^{n+1 / 2}\right)\left(v_{I}^{n+1}-v_{I}^{n}\right)^{2} \\
& +h^{2} \lambda \sum_{n, I} \sum_{\ell}\left(\Theta_{I, \ell}^{+}+\Theta_{I, \ell}^{-}\right) \\
\leq & h^{2} \sum_{I} \eta\left(v_{I}^{0}\right)+2\left\|\eta^{\prime}\right\|_{L^{\infty}} h^{2} \Delta t \sum_{n, I} \frac{1}{h}\left|\sum_{\ell} D_{\ell} g_{\ell}^{\mathrm{EO}}\left(u_{I-e_{\ell} / 2}^{n}, v_{I-e_{\ell}}^{n}, v_{I}^{n}\right)\right| \\
& +C h^{2} \Delta t \sum_{n, I} \sum_{\ell} \frac{1}{h}\left|u_{I+e_{\ell} / 2}^{n}-u_{I-e_{\ell} / 2}^{n}\right| \\
\leq & h^{2} \sum_{I} \eta\left(v_{I}^{0}\right)+C_{\eta^{\prime}}
\end{aligned}
$$

where the constant $C_{\eta^{\prime}}$ depends on the initial variation of $u_{h}$ and the initial divergence bound on g; cf. (3.10).

To get the $L^{2}$ bound, choose $\eta(v)=v^{2}$. Then by an easy lemma in [8],

$$
\begin{aligned}
& \Theta_{I, \ell}^{-} \geq \frac{1}{\left\|\partial_{v} \mathbf{g}\right\|_{L^{\infty}}}\left(D_{\ell}^{v} g_{\ell}^{-}\left(u_{I-e_{\ell} / 2}^{n}, v_{I}^{n}\right)\right)^{2}, \\
& \Theta_{I, \ell}^{+} \geq \frac{1}{\left\|\partial_{v} \mathbf{g}\right\|_{L^{\infty}}}\left(D_{\ell}^{v} g_{\ell}^{+}\left(u_{I-e_{\ell} / 2}^{n}, v_{I-e_{\ell}}^{n}\right)\right)^{2} .
\end{aligned}
$$

Therefore,

$$
\begin{aligned}
& h^{2} \sum_{I}\left(v_{I}^{N}\right)^{2}+h^{2} \sum_{n, I}\left(v_{I}^{n+1}-v_{I}^{n}\right)^{2} \\
& +\frac{\lambda}{\left\|\partial_{v} \mathbf{g}\right\|_{L^{\infty}}} h^{2} \sum_{n, I} \sum_{\ell}\left(D_{\ell}^{v} g_{\ell}^{-}\left(u_{I-e_{\ell} / 2}^{n}, v_{I}^{n}\right)\right)^{2}+\left(D_{\ell}^{v} g_{\ell}^{+}\left(u_{I-e_{\ell} / 2}^{n}, v_{I-e_{\ell}}^{n}\right)\right)^{2} \\
& \quad \leq h^{2} \sum_{I}\left(v_{I}^{0}\right)^{2}+C t^{N} .
\end{aligned}
$$

This has two immediate consequences. Firstly, since

$$
\begin{aligned}
D_{\ell} g_{\ell}^{\mathrm{EO}}\left(u_{I-e_{\ell} / 2}^{n}, v_{I-e_{\ell}}^{n}, v_{I}^{n}\right)= & D_{\ell}^{v} g_{\ell}^{-}\left(u_{I+e_{\ell} / 2}^{n}, v_{I}^{n}\right)+D_{\ell}^{v} g_{\ell}^{+}\left(u_{I+e_{\ell} / 2}^{n}, v_{I-e_{\ell}}^{n}\right) \\
& +D_{\ell}^{u} g_{\ell}^{\mathrm{EO}}\left(u_{I-e_{\ell} / 2}^{n}, v_{I}^{n}, v_{I+e_{\ell}}^{n}\right),
\end{aligned}
$$

we get

$$
\begin{aligned}
\left(D_{\ell} g_{\ell}^{\mathrm{EO}}\right. & \left.\left(u_{I-e_{\ell} / 2}^{n}, v_{I-e_{\ell}}^{n}, v_{I}^{n}\right)\right)^{2} \\
\leq 4 & \left(D_{\ell}^{v} g_{\ell}^{-}\left(u_{I+e_{\ell} / 2}^{n}, v_{I}^{n}\right)\right)^{2}+4\left(D_{\ell}^{v} g_{\ell}^{+}\left(u_{I+e_{\ell} / 2}^{n}, v_{I-e_{\ell}}^{n}\right)\right)^{2} \\
& +2\left(D_{\ell}^{u} g_{\ell}^{\mathrm{EO}}\left(u_{I-e_{\ell} / 2}^{n}, v_{I}^{n}, v_{I+e_{\ell}}^{n}\right)\right)^{2} .
\end{aligned}
$$

Therefore,

$$
\Delta t h^{2} \sum_{n, I} \sum_{\ell}\left(D_{\ell} g_{\ell}^{\mathrm{EO}}\left(u_{I-e_{\ell} / 2}^{n}, v_{I-e_{\ell}}^{n}, v_{I}^{n}\right)\right)^{2} \leq C_{t^{N}} h
$$


where $C_{t}$ is a finite constant depending on $t$ but not on $h$. Secondly, this gives an estimate for the "time variation" of $v_{h}$,

$$
\Delta t h^{2} \sum_{I, n}\left(v_{I}^{n+1}-v_{I}^{n}\right)^{2} \leq C_{t^{N}} h .
$$

We can also bound the variation of the entropy flux. To do this note that

$$
q_{\ell}(u, v)=\mathcal{G}_{\ell}^{+}(u, v)+\mathcal{G}_{\ell}^{-}(u, v),
$$

and by (3.18) and the monotonicity of $g_{\ell}^{ \pm}$,

$$
\begin{aligned}
\left|\mathcal{G}_{\ell}^{ \pm}(u, b)-\mathcal{G}_{\ell}^{ \pm}(u, a)\right| & \leq\left|\eta^{\prime}(b)\right|\left|g_{\ell}^{ \pm}(u, b)-g_{\ell}^{ \pm}(u, a)\right| \\
& +\left|\int_{a}^{b} \eta^{\prime \prime}(s) d s\right|\left|g_{\ell}^{ \pm}(u, b)-g_{\ell}^{ \pm}(u, a)\right| \\
& \leq 3\left\|\eta^{\prime}\right\|_{L^{\infty}}\left|g_{\ell}^{ \pm}(u, b)-g_{\ell}^{ \pm}(u, a)\right| .
\end{aligned}
$$

Thus

$$
\left|q_{\ell}(u, b)-q_{\ell}(u, a)\right| \leq 3\left\|\eta^{\prime}\right\|_{L^{\infty}}\left(\left|g_{\ell}^{+}(u, b)-g_{\ell}^{+}(u, a)\right|+\left|g_{\ell}^{-}(u, b)-g_{\ell}^{-}(u, a)\right|\right) .
$$

Then we arrive at the estimate

$$
\Delta t h^{2} \sum_{I, n} \sum_{\ell}\left(D_{\ell} q_{\ell}\left(u_{I-e_{\ell} / 2}^{n}, v_{I}^{n}\right)\right)^{2} \leq C_{t^{N}} h
$$

Next, let $\left\{w_{h}\right\}_{h>0}$ be a sequence of piecewise constant functions

$$
w_{h}(\mathbf{x}, t)=w_{I}^{n}=\frac{1}{4} \sum_{\ell}\left(u_{I+e_{\ell} / 2}^{n}+u_{I-e_{\ell} / 2}^{n}\right) \quad \text { for } \mathbf{x} \in \mathcal{I}_{i, j} \text { and } t \in\left[t^{n}, t^{n+1}\right) .
$$

Then $w_{h}$ is of bounded variation, i.e.,

$$
\left|w_{h}(\cdot, t)\right|_{B V\left(\mathbb{R}^{2}\right)} \leq C \text { for all } h>0 \text { and for all } t \geq 0 .
$$

Lemma 3.4. Let $\left(\eta_{0}, \mathbf{q}_{0}\right)$ be the Kružkov entropy/entropy flux pair, i.e.,

$$
\eta_{0}(v)=|v-k|, \quad q_{0, \ell}(u, v)=\operatorname{sign}(v-k)\left(g_{\ell}(u, v)-g_{\ell}(u, k)\right),
$$

where $k$ is a constant. Then the sequence

$$
\left\{\partial_{t} \eta_{0}\left(v_{h}\right)+\operatorname{div}\left(\mathbf{q}_{0}\left(w_{h}, v_{h}\right)\right)\right\}_{h}
$$

is compact in $H_{\text {loc }}^{-1}\left(\mathbb{R}^{2} \times \mathbb{R}^{+}\right)$.

Proof. It will be convenient to work with smooth entropies, rather than $\eta_{0}$. Therefore, we let $\eta_{h}$ be a smooth convex approximation to $\eta_{0}$, so that $\eta_{h}(0)=0$ and $\left|\eta_{h}^{\prime}\right| \leq 1$, and we have that

$$
\left\|\eta_{h}-\eta_{0}\right\|_{L^{\infty}} \leq h
$$

Let $\mathbf{q}_{h}$ be the entropy flux corresponding to $\eta_{h}$. Then we also have that

$$
\left\|\mathbf{q}_{h}-\mathbf{q}_{0}\right\|_{L^{\infty}} \rightarrow 0 \quad \text { as } h \rightarrow 0
$$

Let $\varphi$ be a function in $C_{0}^{1}\left(\mathbb{R}^{2} \times \mathbb{R}^{+}\right)$. We use the notation $\hat{\ell}=3-\ell$. Set

$$
\begin{aligned}
\langle\mathcal{L}, \varphi\rangle & =\left\langle\partial_{t} \eta_{0}\left(v_{h}\right)+\operatorname{div}\left(\mathbf{q}_{0}\left(w_{h}, v_{h}\right)\right), \varphi\right\rangle \\
& =\left\langle\mathcal{L}_{h}, \varphi\right\rangle+\left\langle\mathcal{L}-\mathcal{L}_{h}, \varphi\right\rangle,
\end{aligned}
$$

where

$$
\left\langle\mathcal{L}_{h}, \varphi\right\rangle=\left\langle\partial_{t} \eta_{h}\left(v_{h}\right)+\operatorname{div}\left(\mathbf{q}_{0}\left(w_{h}, v_{h}\right)\right), \varphi\right\rangle .
$$


Now

$$
\begin{aligned}
\left|\left\langle\mathcal{L}-\mathcal{L}_{h}, \varphi\right\rangle\right| & \leq \int_{\mathbb{R}^{2} \times \mathbb{R}^{+}}\left|\eta_{h}\left(v_{h}\right)-\eta_{0}\left(v_{h}\right)\right|\left|\varphi_{t}\right| d \mathbf{x} d t \\
& \leq C\left\|\partial_{t} \varphi\right\|_{L^{2}\left(\mathbb{R}^{2} \times \mathbb{R}^{+}\right)}\left\|\eta_{h}-\eta_{0}\right\|_{L^{\infty}} \rightarrow 0 \text { as } h \rightarrow 0
\end{aligned}
$$

where the constant $C$ is independent of $h$ but will depend on the support of $\varphi$. Thus $\mathcal{L}-\mathcal{L}_{h}$ is compact in $H_{\text {loc }}^{-1}\left(\mathbb{R}^{2} \times \mathbb{R}^{+}\right)$.

Now

$$
\begin{aligned}
\left\langle\mathcal{L}_{h}, \varphi\right\rangle= & \left\langle\eta_{h}\left(v_{h}\right)_{t}+\operatorname{div}\left(\mathbf{q}_{0}\left(w_{h}, v_{h}\right)\right), \varphi\right\rangle \\
= & \sum_{n \geq 0} \sum_{I}\left(\eta_{h}\left(v_{I}^{n+1}\right)-\eta_{h}\left(v_{I}^{n}\right)\right) \int_{\mathcal{I}_{I}} \varphi\left(x_{1}, x_{2}, t^{n+1}\right) d \mathbf{x} \\
& +\sum_{n \geq 0} \sum_{I} \sum_{\ell} D_{\ell} q_{0, \ell}\left(w_{I}^{n}, v_{I}^{n}\right) \int_{t^{n}}^{t^{n+1}} \int_{x_{I}-e_{\hat{\ell}} / 2}^{x_{I}+e_{\hat{\ell}} / 2} \varphi\left(x_{I+e_{\ell} / 2}, x_{\hat{\ell}}, t\right) d x_{\hat{\ell}} d t .
\end{aligned}
$$

Set

$$
\varphi_{I}^{n}=\frac{1}{\Delta t h^{2}} \int_{\mathcal{I}_{I}^{n}} \varphi(\mathbf{x}, t) d \mathbf{x} d t
$$

where

$$
\mathcal{I}_{I}^{n}=\mathcal{I}_{I} \times\left[t^{n}, t^{n+1}\right) .
$$

We wish to replace the integrals in the definition of $\mathcal{L}_{h}$ by $h^{2} \varphi_{I}^{n}$ and $h \Delta t \varphi_{I}^{n}$, respectively. The error we make in doing this in the first integral is

$$
\begin{aligned}
& \left|\sum_{n \geq 0, I}\left(\eta_{h}\left(v_{I}^{n+1}\right)-\eta_{h}\left(v_{I}^{n}\right)\right)\left(\int_{\mathcal{I}_{I}} \varphi\left(\mathbf{x}, t^{n+1}\right) d \mathbf{x}-h^{2} \varphi_{I}^{n}\right)\right| \\
& \leq\left\|\eta_{h}^{\prime}\right\|_{L^{\infty}} \sum_{n, I}\left|v_{I}^{n+1}-v_{I}^{n}\right| \frac{1}{\Delta t} \int_{\mathcal{I}_{I}^{n}}\left|\varphi\left(\mathbf{x}, t^{n+1}\right)-\varphi(\mathbf{x}, t)\right| d \mathbf{x} d t \\
& \leq \sum_{n, I}\left|v_{I}^{n+1}-v_{I}^{n}\right| \frac{1}{\Delta t} \int_{\mathcal{I}_{I}^{n}} \int_{t}^{t^{n+1}}\left|\partial_{t} \varphi(\mathbf{x}, s)\right| d s d \mathbf{x} d t \\
& \leq \sum_{n, I}\left|v_{I}^{n+1}-v_{I}^{n}\right| \frac{1}{\Delta t} \int_{\mathcal{I}_{I}} \int_{t^{n}}^{t^{n+1}} \sqrt{t^{n+1}-t}\left(\int_{t^{n}}^{t^{n+1}}\left|\partial_{t} \varphi(\mathbf{x}, s)\right|^{2} d s\right)^{1 / 2} d \mathbf{x} d t \\
& \leq \frac{2}{3} \sum_{n, I}\left|v_{I}^{n+1}-v_{I}^{n}\right| \sqrt{\Delta t} \int_{\mathcal{I}_{I}}\left(\int_{t^{n}}^{t^{n+1}}\left|\partial_{t} \varphi(\mathbf{x}, s)\right|^{2} d s\right)^{1 / 2} d \mathbf{x} \\
& \leq \frac{2}{3} \sum_{n, I}\left|v_{I}^{n+1}-v_{I}^{n}\right| h \sqrt{\Delta t}\left(\int_{\mathcal{I}_{I}^{n}}\left(\partial_{t} \varphi(\mathbf{x}, t)\right)^{2} d \mathbf{x} d t\right)^{1 / 2} \\
& \leq \frac{2}{3}\left(h^{2} \Delta t \sum_{n, I}\left(v_{I}^{n+1}-v_{I}^{n}\right)^{2}\right)^{1 / 2}\left(\sum_{n, I} \int_{\mathcal{I}_{I}^{n}}\left(\partial_{t} \varphi(\mathbf{x}, t)\right)^{2} d \mathbf{x} d t\right)^{1 / 2} \\
& \leq \frac{2}{3} \lambda \sqrt{C_{T} h}\|\varphi\|_{H^{1}\left(\mathbb{R}^{2} \times R^{+}\right)},
\end{aligned}
$$


by (3.23), where $T$ is such that $\varphi(\mathbf{x}, t)=0$ for $t>T$. Next we observe that

$$
\begin{aligned}
& \sum_{\ell}\left|q_{0, \ell}\left(u_{I-e_{\ell} / 2}^{n}, a\right)-q_{0, \ell}\left(w_{I}^{n}, a\right)\right| \\
& \quad \leq \frac{\left\|\partial_{u} \mathbf{q}_{0}\right\|_{L^{\infty}}}{4} \sum_{\ell}\left|u_{I-e_{\ell} / 2}^{n}-u_{I+e_{\ell} / 2}^{n}\right|+\left|u_{I-e_{\ell} / 2}^{n}-u_{I+e_{\hat{\ell}} / 2}^{n}\right|+\left|u_{I-e_{\ell} / 2}^{n}-u_{I-e_{\hat{\ell}} / 2}^{n}\right| .
\end{aligned}
$$

Therefore, the functional $\mathcal{L}_{1}$, defined by

$$
\begin{aligned}
\left\langle\mathcal{L}_{1}, \varphi\right\rangle= & \sum_{n \geq 0} \sum_{I} \sum_{\ell} D_{\ell}\left(q_{0, \ell}\left(w_{I}^{n}, v_{I}^{n}\right)-q_{0, \ell}\left(u_{I-e_{\ell} / 2}^{n}, v_{I}^{n}\right)\right) \\
& \times \int_{t^{n}}^{t^{n+1}} \int_{x_{I}-e_{\hat{\ell}} / 2}^{x_{I}+e_{\hat{\ell}} / 2} \varphi\left(x_{I-e_{\ell} / 2}, x_{\hat{\ell}}, t\right) d x_{\hat{\ell}} d t \\
= & -\sum_{n \geq 0} \sum_{I} \sum_{\ell}\left(q_{0, \ell}\left(w_{I}^{n}, v_{I}^{n}\right)-q_{0, \ell}\left(u_{I-e_{\ell} / 2}^{n}, v_{I}^{n}\right)\right) \int_{\mathcal{I}_{I}^{n}} \partial_{x_{l}} \varphi d \mathbf{x} d t \\
\leq & \sum_{n, I} \sum_{\ell}\left|q_{0, \ell}\left(w_{I}^{n}, v_{I}^{n}\right)-q_{0, \ell}\left(u_{I-e_{\ell} / 2}^{n}, v_{I}^{n}\right)\right| h \sqrt{\Delta t} \\
& \times\left(\int_{\mathcal{I}_{I}^{n}}\left(\partial_{x_{\ell}} \varphi\right)^{2} d \mathbf{x}\right)^{1 / 2} \\
\leq & \left(\sum_{n, I} \sum_{\ell}\left(q_{0, \ell}\left(w_{I}^{n}, v_{I}^{n}\right)-q_{0, \ell}\left(u_{I-e_{\ell} / 2}^{n}, v_{I}^{n}\right)\right)^{2}\right)^{1 / 2}\|\varphi\|_{H^{1}\left(\mathbb{R}^{2} \times \mathbb{R}^{+}\right)} \\
\leq & \sqrt{C T\left|u_{h}(\cdot, 0)\right|_{B V\left(\mathbb{R}^{2}\right)} h}\|\varphi\|_{H^{1}\left(\mathbb{R}^{2} \times \mathbb{R}^{+}\right)},
\end{aligned}
$$

for some constant $C$ that does not depend on $h$. Hence, we can replace $D_{\ell} q_{0, \ell}\left(w_{I}^{n}, v_{I}^{n}\right)$ by $D_{\ell} q_{0, \ell}\left(u_{I-e_{\ell} / 2}^{n}, v_{I}^{n}\right)$ in the second part of $\mathcal{L}_{h}$, making an error which tends to zero in $H_{\text {loc }}^{-1}\left(\mathbb{R}^{2} \times \mathbb{R}^{+}\right)$. Similarly to deriving (3.27), by using (3.24), we get the bound

$$
\begin{aligned}
& \left|\sum_{n, I} \sum_{\ell} D_{\ell} q_{0, \ell}\left(u_{I-e_{\ell} / 2}^{n}, v_{I}^{n}\right)\left(\int_{t^{n}}^{t^{n+1}} \int_{x_{I}-e_{\hat{\ell}} / 2}^{x_{I}+e_{\hat{\ell}} / 2} \varphi\left(x_{I+e_{\ell} / 2}, x_{\hat{\ell}}, t\right) d x_{\hat{\ell}} d t-\Delta t h \varphi_{I}^{n}\right)\right| \\
& \quad \leq \tilde{C}_{T} \sqrt{h}\|\varphi\|_{H^{1}\left(\mathbb{R}^{2} \times R^{+}\right)} .
\end{aligned}
$$

Summing up the discussion so far, we have established that

$$
\begin{aligned}
\langle\mathcal{L}, \varphi\rangle=h^{2} \Delta t \sum_{n, I} & {\left[\frac{1}{\Delta t}\left(\eta_{h}\left(v_{I}^{n+1}\right)-\eta_{h}\left(v_{I}^{n}\right)\right)+\frac{1}{h} \sum_{\ell} D_{\ell} q_{0, \ell}\left(u_{I-e_{\ell} / 2}^{n}, v_{I}^{n}\right)\right] \varphi_{I}^{n} } \\
& + \text { terms which are compact in } H_{\mathrm{loc}}^{-1}\left(\mathbb{R}^{2} \times \mathbb{R}^{+}\right) .
\end{aligned}
$$


By using the "scheme for $\eta$ ", 3.19), we find that the term in square brackets above can be written as

$$
\begin{aligned}
{[\cdots]=} & \underbrace{\frac{1}{\Delta t} \frac{1}{2} \eta_{h}^{\prime \prime}\left(\xi_{I}^{n+1 / 2}\right)\left(v_{I}^{n+1}-v_{I}^{n}\right)^{2}-\frac{1}{h} \sum_{\ell}\left(\Theta_{I, \ell}^{-}+\Theta_{I, \ell}^{+}\right)}_{A_{I}^{n}} \\
& -\underbrace{\frac{1}{h}\left(\eta^{\prime}\left(v_{I}^{n+1}\right)-\eta^{\prime}\left(v_{I}^{n}\right)\right) \sum_{\ell} D_{\ell} g_{\ell}^{\mathrm{EO}}\left(u_{I-e_{\ell} / 2}^{n}, v_{I-e_{\ell}}^{n}, v_{I}^{n}\right)}_{B_{I}^{n}} \\
& -\underbrace{\frac{1}{h} \sum_{\ell} D_{\ell}^{u}\left(\eta^{\prime}\left(v_{I}^{n}\right) g_{\ell}^{\mathrm{EO}}\left(u_{I-e_{\ell} / 2}^{n}, v_{I}^{n}, v_{I+e_{\ell}}^{n}\right)-q_{\ell}^{\mathrm{EO}}\left(u_{I-e_{\ell} / 2}^{n}, v_{I}^{n}, v_{I+e_{\ell}}^{n}\right)\right)}_{C_{I}^{n}} \\
& -\underbrace{\frac{1}{h} \sum_{\ell} D_{\ell}\left(q_{\ell}^{\mathrm{EO}}\left(u_{I-e_{\ell} / 2}^{n}, v_{I-e_{\ell}}^{n}, v_{I}^{n}\right)-q_{0, \ell}\left(u_{I-e_{\ell} / 2}^{n}, v_{I}^{n}\right)\right)}_{D_{I}^{n}} .
\end{aligned}
$$

Thus we can write

$$
\langle\mathcal{L}, \varphi\rangle=\langle\mathcal{A}, \varphi\rangle+\langle\mathcal{B}, \varphi\rangle+\langle\mathcal{C}, \varphi\rangle+\langle\mathcal{D}, \varphi\rangle+\text { compact terms }
$$

with

$$
\begin{gathered}
\langle\mathcal{A}, \varphi\rangle=h^{2} \Delta t \sum_{n, I} A_{I}^{n} \varphi_{I}^{n}, \quad\langle\mathcal{B}, \varphi\rangle=h^{2} \Delta t \sum_{n, I} B_{I}^{n} \varphi_{I}^{n}, \\
\langle\mathcal{C}, \varphi\rangle=h^{2} \Delta t \sum_{n, I} C_{I}^{n} \varphi_{I}^{n} \quad \text { and } \quad\langle\mathcal{D}, \varphi\rangle=h^{2} \Delta t \sum_{n, I} D_{I}^{n} \varphi_{I}^{n} .
\end{gathered}
$$

By (3.20),

$$
\begin{aligned}
\langle\mathcal{A}, \varphi\rangle & \leq\|\varphi\|_{L^{\infty}\left(\mathbb{R}^{2} \times \mathbb{R}\right)}\left(\frac{h^{2}}{2} \sum_{n, I} \eta_{h}^{\prime \prime}\left(\xi_{I}^{n+1 / 2}\right)\left(v_{I}^{n+1}-v_{I}^{n}\right)^{2}+h \Delta t \sum_{n, I} \sum_{\ell}\left(\Theta_{I, \ell}^{-}+\Theta_{I, \ell}^{+}\right)\right) \\
& \leq C_{T}\|\varphi\|_{L^{\infty}\left(\mathbb{R}^{2} \times \mathbb{R}^{+}\right)} .
\end{aligned}
$$

Therefore, $\mathcal{A} \in \mathcal{M}_{\text {loc }}\left(\mathbb{R}^{2} \times \mathbb{R}^{+}\right)$. By the divergence bound on the numerical flux (3.10) and the $B V$ bound on $u_{h}$,

$$
|\langle\mathcal{B}+\mathcal{C}, \varphi\rangle| \leq C\|\varphi\|_{L^{\infty}\left(\mathbb{R}^{2} \times \mathbb{R}^{+}\right)} ;
$$

thus also $\mathcal{B}+\mathcal{C} \in \mathcal{M}_{\text {loc }}\left(\mathbb{R}^{2} \times \mathbb{R}^{+}\right)$.

To estimate $\mathcal{D}$ we start by observing that for $\varepsilon>0$,

$$
\begin{aligned}
q_{\varepsilon, \ell}^{\mathrm{EO}}(u, a, b)-q_{\varepsilon, \ell}(u, b)= & q_{\varepsilon, \ell}^{\mathrm{EO}}(u, a, b)-q_{\varepsilon, \ell}^{\mathrm{EO}}(b, b) \\
= & \int_{b}^{a} \eta_{\varepsilon}^{\prime}(s) \partial_{v} g^{-}(u, s) d s \\
= & \eta_{\varepsilon}^{\prime}(a)\left(g^{-}(u, a)-g^{-}(u, b)\right) \\
& -\int_{b}^{a} \eta_{\varepsilon}^{\prime \prime}(s)\left(g^{-}(u, s)-g^{-}(u, a)\right) d s .
\end{aligned}
$$

As before, by using the monotonicity of $g^{-}$we get $\left|q_{\varepsilon, \ell}^{\mathrm{EO}}(u, a, b)-q_{\varepsilon, \ell}(u, b)\right| \leq 3\left\|\eta_{\varepsilon}^{\prime}\right\|_{L^{\infty}}\left|g^{-}(u, a)-g^{-}(u, b)\right| \leq 3\left|g^{-}(u, a)-g^{-}(u, b)\right|$. 
Sending $\varepsilon$ to zero gives

$$
\left|q_{0, \ell}^{\mathrm{EO}}(u, a, b)-q_{0, \ell}(u, b)\right| \leq 3\left|g^{-}(u, a)-g^{-}(u, b)\right| .
$$

Next, by a partial summation,

$$
\begin{aligned}
& \left|h^{2} \Delta t \sum_{n, I} D_{I}^{n} \varphi_{I}^{n}\right|=\mid h^{2} \Delta t \sum_{n, I} \sum_{\ell}\left(g_{\ell}^{\mathrm{EO}}\left(u_{I-e_{\ell} / 2}^{n}, v_{I-e_{\ell}}^{n}, v_{I}^{n}\right)\right. \\
& \left.\quad-q_{0, \ell}\left(u_{I-e_{\ell} / 2}^{n}, v_{I}^{n}\right)\right) \frac{1}{h} D_{\ell} \varphi_{I-e_{\ell}}^{n} \mid \\
& \quad \leq 3 \sum_{\ell} h^{2} \Delta t \sum_{n, I}\left|D_{\ell}^{v} g^{-}\left(u_{I-e_{\ell} / 2}^{n}, v_{I-e_{\ell}}^{n}\right)\right| \frac{1}{h}\left|D_{\ell} \varphi_{I}^{n}\right| \\
& \quad \leq 3 \sum_{\ell} \sqrt{h^{2} \Delta t \sum_{n, I}\left(D_{\ell}^{v} g^{-}\left(u_{I-e_{\ell} / 2}^{n}, v_{I-e_{\ell}}^{n}\right)\right)^{2}} \sqrt{h^{2} \Delta t \sum_{n, I}\left(\frac{1}{h}\left|D_{\ell} \varphi_{I}^{n}\right|\right)^{2}} .
\end{aligned}
$$

Using the bound (3.21) (for $g^{-}$) we find that

$$
|\langle\mathcal{C}, \varphi\rangle| \leq C_{T} \sqrt{h} \sum_{\ell}\left\|\partial_{x_{\ell}} \varphi\right\|_{L^{2}\left(\mathbb{R}^{2} \times \mathbb{R}^{+}\right)} .
$$

Therefore, $\mathcal{D}$ is compact in $H_{\mathrm{loc}}^{-1}\left(\mathbb{R}^{2} \times \mathbb{R}^{+}\right)$.

Hence we have established that $\mathcal{L}$ is compact in $H_{\text {loc }}^{-1}\left(\mathbb{R}^{2} \times \mathbb{R}^{+}\right)$.

Since $u_{h}$ converges strongly in $L^{p}$ to $u$, and $u_{h}$ is of bounded variation, also $w_{h}$ will converge strongly to $u$ in $L^{p}$ for any $p \in[1, \infty)$. In particular this implies

$$
\left|\left\langle\operatorname{div}\left(\mathbf{q}_{0}\left(w_{h}, v_{h}\right)-\mathbf{q}\left(u, v_{h}\right)\right), \varphi\right\rangle\right| \leq C\left\|w_{h}-u\right\|_{L^{2}\left(\mathbb{R}^{2} \times \mathbb{R}^{+}\right)}\|\varphi\|_{H^{1}\left(\mathbb{R}^{2} \times \mathbb{R}^{+}\right)} \rightarrow 0,
$$

as $h \rightarrow 0$. Thus the sequence

$$
\left\{\partial_{t} \eta_{0}\left(v_{h}\right)+\operatorname{div}\left(\mathbf{q}_{0}\left(u, v_{h}\right)\right)\right\}_{h>0}
$$

is compact in $H_{\text {loc }}^{-1}\left(\mathbb{R}^{2}\right)$. Therefore, by Lemma 2.2 there exists a subsequence $\left\{v_{h}\right\}$ (which we do not relabel) and a function $v \in L^{\infty}\left(\mathbb{R}^{2} \times \mathbb{R}^{+}\right)$such that

$$
v_{h} \rightarrow v \quad \text { a.e. and in } L_{\mathrm{loc}}^{p}\left(\mathbb{R}^{2} \times \mathbb{R}^{+}\right) \text {, for any } p \in[1, \infty) \text {. }
$$

Lemma 3.5. Let $u$ and $v$ be the maps defined in (3.4) and (3.31), respectively. The pair $(u, v)$ is a weak solution of the Cauchy problem (1.1)-(1.2) in the sense of Definition 2.1.

Proof. Recalling Lemma 3.1 we only have to verify that $v$ is a weak solution of the conservation law (1.2), i.e., that the second equation in Definition 2.1 holds.

Define

$$
D_{t} v_{I}^{n}=v_{I}^{n+1}-v_{I}^{n}
$$

With this notation the scheme for $v$, (3.11), can be written as

$$
\frac{1}{\Delta t} D_{t} v_{I}^{n}+\sum_{\ell} \frac{1}{h} D_{\ell} g_{\ell}^{\mathrm{EO}}\left(u_{I-e_{\ell} / 2}^{n}, v_{I-e_{\ell}}^{n}, v_{I}^{n}\right)=0 .
$$


Let $\varphi \in C_{0}^{\infty}\left(\mathbb{R}^{2} \times \mathbb{R}_{0}^{+}\right)$be a test function and let $\varphi_{I}^{n}$ be defined by (3.26). Multiplying the above by $h^{2} \Delta t \varphi_{I}^{n}$ and doing a partial summation, we find that

$$
\begin{aligned}
0=\underbrace{h^{2} \sum_{I} v_{I}^{0} \varphi_{I}^{0}}_{I_{0}}+\underbrace{h^{2} \Delta t \sum_{n \geq 1, I} v_{I}^{n} \frac{1}{\Delta t} D_{t} \varphi_{I}^{n-1}}_{I_{1}} \\
+\underbrace{h^{2} \Delta t \sum_{n, I} \sum_{\ell} g_{\ell}^{\mathrm{EO}}\left(u_{I+e_{\ell} / 2}^{n}, v_{I}^{n}, v_{I+e_{\ell}}^{n}\right) \frac{1}{h} D_{\ell} \varphi_{I}^{n}}_{I_{2}}
\end{aligned}
$$

Now

$$
I_{0}=\int_{\mathbb{R}^{2}} v_{h}(\mathbf{x}, 0) \varphi(\mathbf{x}, 0) d \mathbf{x}+\underbrace{\sum_{I} v_{I}^{0} \int_{\mathcal{I}_{I}} \int_{0}^{\Delta t} \frac{\varphi(\mathbf{x}, s)-\varphi(\mathbf{x}, 0)}{\Delta t} d s d \mathbf{x}}_{I_{0,1}}
$$

The term $I_{0,1}$ can be estimated by observing that

$$
\begin{aligned}
\left|I_{0,1}\right| & \leq \sum_{I}\left|v_{I}^{0}\right| \int_{\mathcal{I}_{I}} \int_{0}^{\Delta t} \frac{1}{\Delta t} \int_{0}^{s} \partial_{t} \varphi(\mathbf{x}, \tau) d \tau d s d \mathbf{x} \\
& \leq \sum_{I}\left|v_{I}^{0}\right| \int_{\mathcal{I}_{I}} \frac{\left\|\partial_{t} \varphi\right\|_{L^{\infty}}}{\Delta t} \int_{0}^{\Delta t} s d s d \mathbf{x} \\
& \leq \frac{\left\|\partial_{t} \varphi\right\|_{L^{\infty}} \Delta t}{2}\left\|v_{0}\right\|_{L^{1}\left(\mathbb{R}^{2}\right)} \rightarrow 0 \quad \text { as } \Delta t \rightarrow 0 .
\end{aligned}
$$

Using this and the bounded convergence theorem,

$$
\lim _{h \rightarrow 0} I_{0}=\int_{\mathbb{R}^{2}} v_{0}(\mathbf{x}) \varphi(\mathbf{x}, 0) d \mathbf{x}
$$

Similarly to the estimate on $I_{0}$, we can write $I_{1}$ as

$$
I_{1}=\int_{\Delta t}^{\infty} \int_{\mathbb{R}^{2}} v_{h}(\mathbf{x}, t) \partial_{t} \varphi(\mathbf{x}, t) d \mathbf{x} d t+\underbrace{\sum_{n \geq 1, I} v_{I}^{n} \int_{\mathcal{I}_{I}^{n}} \int_{t-\Delta t}^{t} \frac{\partial_{t} \varphi(\mathbf{x}, s)-\partial_{t} \varphi(\mathbf{x}, t)}{\Delta t} d s d \mathbf{x} d t}_{I_{1,1}}
$$

where $I_{1,1}$ can be bounded as

$$
\left|I_{1,1}\right| \leq \Delta t\left\|\partial_{t t} \varphi\right\|_{L^{\infty}}\left\|v_{h}(\cdot, t)\right\|_{L^{1}(\mathbb{R})} \rightarrow 0 \quad \text { as } \Delta t \rightarrow 0 .
$$


Therefore,

$$
\lim _{h \rightarrow 0} I_{1}=\int_{0}^{\infty} \int_{\mathbb{R}^{2}} v \partial_{t} \varphi d \mathbf{x} d t
$$

The term $I_{2}$ is slightly more complicated. Writing $I_{2}=\sum_{\ell} I_{2}^{\ell}$ we get

$$
\begin{aligned}
I_{2}^{\ell}=\int_{0}^{\infty} & \int_{\mathbb{R}^{2}} g\left(w_{h}, v_{h}\right) \partial_{x_{\ell}} \varphi d \mathbf{x} d t \\
& -\underbrace{\sum_{n, I} g\left(w_{I}^{n}, v_{I}^{n}\right) \int_{\mathcal{I}_{I}^{n}} \int_{0}^{h} \frac{\partial_{x_{\ell}} \varphi(\mathbf{x}, t)-\partial_{x_{\ell}} \varphi\left(\mathbf{x}+x e_{\ell}\right)}{h} d x d \mathbf{x} d t}_{I_{2,1}^{\ell}} \\
& -\underbrace{h^{2} \Delta t \sum_{n, I}\left(g\left(w_{I}^{n}, v_{I}^{n}\right)-g\left(u_{I+e_{\ell} / 2}^{n}, v_{I}^{n}\right)\right) \frac{1}{h} D_{\ell} \varphi_{I}^{n}}_{I_{2,2}^{\ell}} \\
& -\underbrace{h^{2} \Delta t \sum_{n, I}\left(g_{\ell}^{\mathrm{EO}}\left(u_{I+e_{\ell} / 2}^{n}, v_{I}^{n}, v_{I}^{n}\right)-g_{\ell}^{\mathrm{EO}}\left(u_{I+e_{\ell} / 2}^{n}, v_{I}^{n}, v_{I+e_{\ell}}^{n}\right)\right) \frac{1}{h} D_{\ell} \varphi_{I}^{n}}_{I_{2,3}^{\ell}} .
\end{aligned}
$$

The term $I_{2,1}^{\ell}$ can be estimated as $I_{1,1}$,

$$
\left|I_{2,1}^{\ell}\right| \leq h\left\|\partial_{x_{\ell} x_{\ell}} \varphi\right\|_{L^{\infty}}\left\|g\left(w_{h}, v_{h}\right)\right\|_{L^{1}\left([-M, M]^{2} \times[0, T]\right)} \rightarrow 0 \quad \text { as } h \rightarrow 0,
$$

where $M$ and $T$ are such that $\varphi(x, t)=0$ for $t>T$ and $|\mathbf{x}|>M$. Since $u_{h}$ and $w_{h}$ are of bounded variation,

$$
\left|I_{2,2}^{\ell}\right| \leq C\left|u_{0}\right|_{B V\left(\mathbb{R}^{2}\right)}\left\|\partial_{x_{\ell}} \varphi\right\|_{L^{\infty}} h \rightarrow 0 \quad \text { as } h \rightarrow 0,
$$

for some constant $C$ which is independent of $h$. For the final term we use (3.21) and write

$$
\begin{aligned}
\left|I_{2,3}^{\ell}\right| & =h^{2} \Delta t \sum_{n, I} D_{\ell}^{v} g_{\ell}^{+}\left(u_{I+e_{\ell} / 2}^{n}, v_{I}^{n}\right) \frac{1}{h} D_{\ell} \varphi_{I}^{n} \\
& \leq\left(h^{2} \Delta t \sum_{n, I}\left(D_{\ell}^{v} g_{\ell}^{+}\left(u_{I+e_{\ell} / 2}^{n}, v_{I}^{n}\right)\right)^{2}\right)^{1 / 2}\left(h^{2} \Delta t \sum_{n, I}\left(\frac{D_{\ell} \varphi_{I}^{n}}{h}\right)^{2}\right)^{1 / 2} \\
& \leq \sqrt{C_{T} h}\left\|\partial_{x} \varphi\right\|_{L^{2}\left(\mathbb{R}^{2} \times \mathbb{R}^{+}\right)} \rightarrow 0 \quad \text { as } h \rightarrow 0 .
\end{aligned}
$$

Collecting the bounds (3.34), (3.35) and (3.36) and using the strong convergence of $w_{h}$ and $v_{h}$ we find that

$$
\lim _{h \rightarrow 0} I_{2}=\int_{0}^{\infty} \int_{\mathbb{R}^{2}} \mathbf{g}(u, v) \operatorname{div}(\varphi) d \mathbf{x} d t
$$

Having the limits (3.32), (3.33) and (3.37) we observe that the limit $v$ is a weak solution. 


\section{NUMERICAL EXAMPLES}

We close this paper by demonstrating how this scheme works in practice. In the first example we approximate solutions of the equation

$$
\begin{aligned}
\partial_{t} u+\partial_{x}\left(\frac{1}{2}\left(u^{2}\right)\right)+\partial_{y}\left(\frac{1}{2} u\right) & =0, \\
\partial_{t} v+\partial_{x}(u v(1-v))+\partial_{y}(v(v+u)) & =0,
\end{aligned}
$$

with initial data

$$
u(x, y, 0)=2 e^{-4\left(x^{2}+y^{2}\right)}-1 \quad \text { and } \quad v(x, y, 0)=\frac{1}{2}(1-\sin (\pi x)),
$$

for $(x, y) \in[-1,1]^{2}$ and periodically extended outside this square. In Figure 2 we show the approximations $u_{h}$ and $v_{h}$ for $t=0.5$ computed on a grid with $h=1 / 128$. The $v$ variable is depicted as a colored plot, and $u$ as superposed contours.

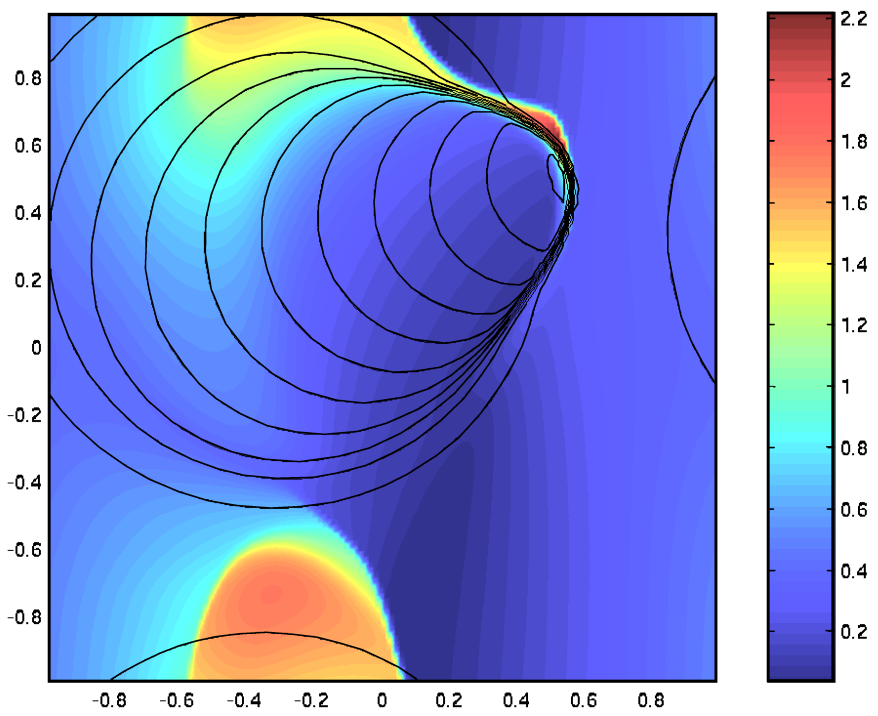

Figure 2. The approximate solution of (4.1)-(4.3) at $t=1 / 2$.

As a second example, we use a system of equations based on a model of threephase flow in porous media as mentioned in the introduction. Assume that the porous medium is two-dimensional and that gravity acts in the $y$-direction. Since we are concerned with the saturation equations, instead of letting the Darcy velocity be defined as the solution of the elliptic equation (1.4), we fix a velocity field $U=(1,0)$. This is supposed to mimic the situation where we are injecting at the left boundary and extracting along the right boundary. Let $u$ and $v$ denote the gas and water saturations, respectively. In this setting, the equations (1.5) take the form

$$
\begin{aligned}
\partial_{t} u+\partial_{x} f_{1}(u, v)+\partial_{y} f_{2}(u, v) & =0, \\
\partial_{t} v+\partial_{x} g_{1}(u, v)+\partial_{y} g_{2}(u, v) & =0,
\end{aligned}
$$


where

$$
\begin{aligned}
& f_{1}(u, v)=\frac{\lambda_{\mathrm{g}}(u)}{\lambda_{\mathrm{T}}(u, v)}, \\
& f_{2}(u, v)=k \frac{\lambda_{\mathrm{g}}(u)}{\lambda_{\mathrm{T}}(u, v)}\left(\lambda_{\mathrm{w}}(v)\left(\rho_{\mathrm{g}}-\rho_{\mathrm{w}}\right)+\lambda_{\mathrm{o}}(1-u-v)\left(\rho_{\mathrm{g}}-\rho_{\mathrm{o}}\right)\right), \\
& g_{1}(u, v)=\frac{\lambda_{\mathrm{w}}(v)}{\lambda_{\mathrm{T}}(u, v)}, \\
& g_{2}(u, v)=k \frac{\lambda_{\mathrm{w}}(v)}{\lambda_{\mathrm{T}}(u, v)}\left(\lambda_{\mathrm{g}}(u)\left(\rho_{\mathrm{w}}-\rho_{\mathrm{g}}\right)+\lambda_{\mathrm{o}}(1-u-v)\left(\rho_{\mathrm{w}}-\rho_{\mathrm{o}}\right)\right),
\end{aligned}
$$

where

$$
\lambda_{\mathrm{g}}(u)=\frac{u^{2}}{\nu_{\mathrm{g}}}, \quad \lambda_{\mathrm{w}}(v)=\frac{v^{2}}{\nu_{\mathrm{w}}}, \quad \lambda_{\mathrm{o}}(u)=\frac{w^{2}}{\nu_{\mathrm{o}}}, \quad \text { and } \quad \lambda_{\mathrm{T}}=\lambda_{\mathrm{g}}+\lambda_{\mathrm{w}}+\lambda_{\mathrm{o}} .
$$

The model (4.4) is not a triangular model, but often the viscosity of the gaseous phase is much smaller than the viscosities of oil and water. Thus, "from the gas' point of view", oil and water are very similar, or $f_{i}(u, v) \approx f_{i}(u,(1-u) / 2)$. Hence, setting $v=(1-u) / 2$ in the first equation in (4.4) should be a reasonable approximation. This yields the triangular model (1.7). In the second example we therefore use (4.4) with $f_{i}(u, v)$ replaced by $f_{i}(u,(1-u) / 2)$, with the parameter values

$$
\begin{gathered}
\nu_{\mathrm{g}}=1, \quad \nu_{\mathrm{w}}=80, \quad \nu_{\mathrm{o}}=100 \\
\rho_{\mathrm{g}}=0.05, \quad \rho_{\mathrm{w}}=1.00, \quad \rho_{\mathrm{o}}=0.85, \quad \text { and } \quad k=500 .
\end{gathered}
$$

We used the computational domain $(x, y) \in[0,1]^{2}$, and the initial values are

$$
u(x, 0)=\left\{\begin{array}{ll}
0 & \text { if } x<0.1, \\
e^{y-1} & \text { otherwise }
\end{array} \quad v(x, 0)= \begin{cases}1 & \text { if } x<0.1 \\
0 & \text { otherwise }\end{cases}\right.
$$

The system (4.4) with these initial values can be viewed as a simplistic model of water injection in a porous medium filled with oil and gas, where most of the lighter gas is on top of the oil. Recall that the velocity in (4.4) is fixed. Nevertheless, if one uses a sequential method to solve the full system, the hyperbolic part of the model will be of the type (4.4).

In Figure 3 we show the time evolution of the three phases for $t \in[0,0.4]$. We have computed the approximate solution using $h=1 / 128$ and the boundary conditions

$$
\begin{gathered}
u(0, y, t)=0, \quad v(0, y, t)=1, \quad \mathbf{f}=\mathbf{g}=0 \quad \text { for } y=0 \text { and } y=1, \\
\partial_{x} u=\partial_{x} v=0 \quad \text { on } x=1 .
\end{gathered}
$$



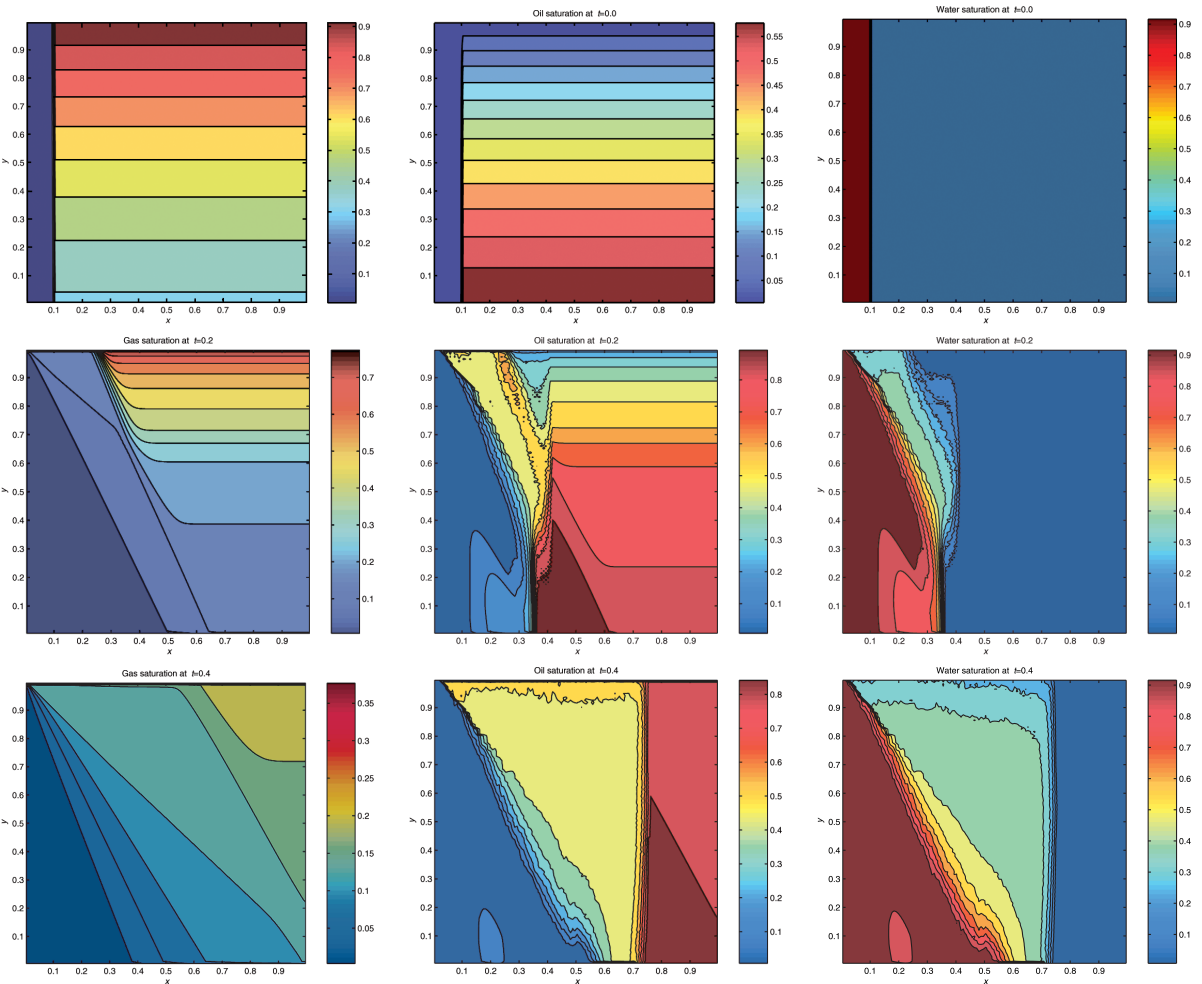

Figure 3 . The saturations at $t=0$ (top), $t=0.2$ (middle) and $t=$ 0.4 (bottom). Left column: gas $(u)$, middle column: oil $(1-u-v)$, right column: water $(v)$.

\section{REFERENCES}

1. Adimurthi, S. Mishra and G. D. V. Gowda. Optimal entropy solutions for conservation laws with discontinuous flux-functions. J. Hyperbolic Differ. Equ., 2 (4), 783-837, 2005. MR.2195983 (2007g:35144)

2. S. Benzoni-Gavage and D. Serre. Multidimensional hyperbolic partial differential equations, First-order systems and applications. Oxford Mathematical Monographs. The Clarendon Press, Oxford University Press, Oxford, 2007. MR.2284507 (2008k:35002)

3. R. Bürger, K.H. Karlsen, N.H. Risebro and J.D. Towers. Well-posedness in $B V_{t}$ and convergence of a difference scheme for continuous sedimentation in ideal clarifier-thickener units. Numer. Math., 97 (1):25-65, 2004. MR2045458 (2004m:35175)

4. Z. Chen, G. Huan and Y. Ma. Computational methods for multiphase flows in porous media. Computational Science and Engineering, SIAM, 2006. MR2217767 (2007c:76070)

5. G. M. Coclite, K. H. Karlsen, S. Mishra and N. H. Risebro. Convergence of the viscous approximation for a triangular system of conservation laws. To appear in Unione Mat. Ital.

6. M. G. Crandall and L. Tartar. Some relations between nonexpansive and order preserving mappings. Proc. Amer. Math. Soc 78 (3), 385 - 390, 1980. MR.553381 (81a:47054)

7. S. Diehl. A conservation law with point source and discontinuous flux function modelling continuous sedimentation. SIAM J. Appl. Math., 56 (2):1980-2007, 1995. MR.1381652(97a:35145)

8. R. Eymard, T. Gallouet, M. Ghilani and R. Herbin. Error estimates for the approximate solutions of nonlinear conservation laws given by finite difference schemes. IMA J. Numer. Anal., 18(4), 563-594, 1998. MR1681074 (2000b:65180) 
9. T. Gimse and N. H. Risebro. Solution of Cauchy problem for a conservation law with discontinuous flux function. SIAM J. Math. Anal, 23 (3): 635-648, 1992. MR.1158825 (93e:35070)

10. H. Holden and N. H. Risebro. Front tracking for hyperbolic conservation laws, volume 152 of Applied Mathematical Sciences. Springer-Verlag, New York, 2002. MR.1912206 (2003e:35001)

11. L. Holden. On the strict hyperbolicity of the Buckley-Leverett equations for three-phase flow in a porous medium. SIAM J. Appl. Math., 50 (3), 667-682, 1990. MR1050906 (91c:35098)

12. L. Hörmander. Lectures on nonlinear hyperbolic differential equations. Mathematics and Applications, Vol. 26, Springer, 1997. MR.1466700 (98e:35103)

13. K. H. Karlsen, S. Mishra and N. H. Risebro. Convergence of finite volume schemes for triangular systems of conservation laws. Numer. Math., 111(4), 2009, 559-589. MR2471610

14. K. H. Karlsen, M. Rascle and E. Tadmor. On the existence and compactness of a twodimensional resonant system of conservation laws. Commun. Math. Sci, 5(2), 2007, 253-265. MR 2334842(2008j:35121)

15. K. H. Karlsen, N.H. Risebro and J.D. Towers. $L^{1}$ stability for entropy solutions of nonlinear degenerate parabolic convection-diffusion equations with discontinuous coefficients. Skr. K. Nor. Vidensk. Selsk.,3, 2003, 49 pages. MR2024741 (2004j:35149)

16. K. H. Karlsen and N. H. Risebro. Convergence of finite difference schemes for viscous and inviscid conservation laws with rough coefficients. M2AN Math. Model. Numer. Anal., 35 (2), 239-269, 2001. MR 1825698(2002b:35138)

17. K. H. Karlsen and J. D. Towers. Convergence of the Lax-Friedrichs scheme and stability for conservation laws with a discontinous space-time dependent flux. Chinese Ann. Math. Ser. B, 25(3):287-318, 2004. MR2086124(2005h:65145)

18. F. Murat. L'injection du cône positif de $H^{-1}$ dans $W^{-1, q}$ est compacte pour tout $q<2 . J$. Math. Pures Appl. (9), 60(3):309-322, 1981. MR633007 (83b:46045)

19. E. Yu. Panov. Existence and strong pre-compactness properties for entropy solutions of a first-order quasilinear equation with discontinuous flux. Submitted.

20. J.D. Towers. Convergence of a difference scheme for conservation laws with a discontinuous flux. SIAM J. Numer. Anal., 38(2):681-698, 2000. MR.1770068 (2001f:65098)

21. J.D. Towers. A difference scheme for conservation laws with a discontinuous flux: the nonconvex case. SIAM J. Numer. Anal., 39(4): 1197-1218, 2001. MR.1870839 (2002k:65131)

Department of Mathematics, University of Bari, via E. Orabona 4, I-70125 Bari, ITALY

E-mail address: coclitegm@dm.uniba.it

URL: http://www.dm.uniba.it/Members/coclitegm

Centre of Mathematics for Applications (CMA), University of Oslo, P.O. Box 1053, Blindern, N-0316 Oslo, Norway

E-mail address: siddharm@math.uio.no

URL: http://folk.uio.no/siddharm

Centre of Mathematics for Applications (CMA), University of Oslo, P.O. Box 1053, Blindern, N-0316 OSLO, NoRWAy

E-mail address: nilshr@math.uio.no

URL: http://www.math.uio.no/〜nilshr/ 